\title{
Article \\ A Numerical Method to Improve the Representativeness of Real Microstructure Cut-Outs Applied in Finite Element Simulations
}

\author{
Yanling Schneider ${ }^{1, *} \mathbb{\infty}$, Werner Wasserbäch ${ }^{2}$, Siegfried Schmauder ${ }^{1}\left(\mathbb{D}\right.$, Zhangjian Zhou $^{3}$, Reiner Zielke ${ }^{4}$ \\ and Wolfgang Tillmann ${ }^{4}(\mathbb{D}$
}

1 Institute for Materials Testing, Materials Science and Strength of Materials, University of Stuttgart, Pfaffenwaldring 32, D-70569 Stuttgart, Germany; Siegfried.Schmauder@imwf.uni-stuttgart.de

2 Institute of Materials Science, University of Stuttgart, Heisenbergstraße 3, D-70569 Stuttgart, Germany; Werner.Wasserbaech@imw.uni-stuttgart.de

3 School of Materials Science and Engineering, University of Science and Technology Beijing, Beijing 100083, China; ZhouZhj@mater.ustb.edu.cn

4 RIF Institute for Research and Transfer e.V., Joseph-von-Fraunhofer Str. 20, D-44227 Dortmund, Germany; Reiner.Zielke@tu-dortmund.de (R.Z.); Wolfgang.Tillmann@tu-dortmund.de (W.T.)

* Correspondence: yanling.schneider@imwf.uni-stuttgart.de

Citation: Schneider, Y.; Wasserbäch, W.; Schmauder, S.; Zhou, Z.; Zielke,

R.; Tillmann, W. A Numerical

Method to Improve the

Representativeness of Real

Microstructure Cut-Outs Applied in

Finite Element Simulations. Crystals 2021, 11, 382. https://doi.org/

$10.3390 /$ cryst11040382

Academic Editors: Cyril Cayron,

Napat Vajragupta, Junhe Lian and

Sebastian Münstermann

Received: 19 February 2021

Accepted: 30 March 2021

Published: 6 April 2021

Publisher's Note: MDPI stays neutral with regard to jurisdictional claims in published maps and institutional affiliations.

Copyright: (c) 2021 by the authors. Licensee MDPI, Basel, Switzerland. This article is an open access article distributed under the terms and conditions of the Creative Commons Attribution (CC BY) license (https:// creativecommons.org/licenses/by/ $4.0 /)$.

\begin{abstract}
To improve the representativeness of a real microstructural cut-out for modeling purposes, a numerical method named as "boundary pixel color alteration (BPCA)" is presented to modify measured 2D microstructure cut-outs. Its physical background is related to the phase growth. For the application, the precondition is that the representativeness of the microstructure is already satisfied to a certain extent. This method resolves the problem that the phase composition of a small cut-out can have a large discrepancy to the real one. The main idea is to change the pixel color among neighboring pixels belonging to different phases. Our process simultaneously maintains most of the characteristics of the original morphology and is applicable for nearly all kinds of multi-phase or polycrystalline metallic alloys, as well. From our axisymmetric finite element (FE) simulations (ABAQUS ) applied with 2D real microstructures, it shows that the volume ratios of microstructural phases, as a function of the structure position to the symmetric axis, converge to phase area ratios in the $2 \mathrm{D}$ cut-out, even though the axisymmetric element volume is position dependent. A mathematical proof provides the reason for the aforementioned convergence. As examples to achieve real compositions and to numerically prove the aforementioned convergence, four different materials including multiphase polycrystals are implemented. An improvement of the predicted FE result is presented for the application of a modified microstructure (with a higher representativeness) compared to the original one.
\end{abstract}

Keywords: multi-phase polycrystalline material; real composition; microstructure representativeness; boundary pixel color alteration; micromechanical FE simulation

\section{Introduction}

Metals and alloys are important construction materials. To guarantee the structure safety against loading, it is essential to have a thorough understanding of their deformation behavior. Experimental measurements and numerical approaches are two common ways to obtain details of the aforementioned deformation behavior. The knowledge of microstructural characteristics is important for material deformation investigations. To achieve such knowledge relies upon experimental techniques of non-destructive and destructive nature. Such measurements can be, e.g., transmission electron microscopy (TEM), X-ray diffraction (XRD), and electron backscatter diffraction (EBSD). One application of indentation testing can be found in Lasko et al. [1], which investigated the mechanical properties of bio-inspired $\mathrm{TiO}_{2}$ / organic-polyelectrolyte-layered nano composites. By using the EBSD technique, it is possible to evaluate the grain structure and the inclusion of metal matrix 
composites (MMCs). Such evaluations cover, e.g., grain crystallographic orientations and textures, as well as additive sizes and distributions. Recently, 3D EBSD has already been applied in investigations of material behavior [2-4]. For a relatively comprehensive description of the EBSD method, it can be referred to, e.g., Engler and Randle [5]. Concerning numerical approaches/simulations, the finite element method (FEM) is the most commonly used method today. By using one-scale, two-scale or multi-scale FE simulations, it is possible to describe different deformation modes under static and dynamic loadings. For a detailed study of the material deformation behavior, the application of the real microstructure is preferred in FE simulations. Such numerical calculations can include different mechanical and physical influential factors, e.g., the crystallographic orientation of grains, the local interaction between grain-grain and grain-particle, and the local inhomogeneity in the matrix and the inclusion. When a real or an artificial microstructure is applied in an FE analysis, its representativeness is important and should be considered with respect to, e.g., the phase volume fraction, the mean size and distribution of each phase. Even though the study of the microstructure representativeness has already existed for a few decades, it is still not fully accomplished. In order to statistically represent the (artificial) microstructure, Mirkhalaf et al. [6] proposed a methodology based on statistical analysis and numerical experiments to determine the size of the representative volume element (RVE). In particular, for individual applications of real microstructures, specific solutions may be necessary to improve the representativeness. This representativeness can be weakened due to some possible problems, e.g., the total grain number is statistically not enough to present the texture; the mean particle size in the cut-out has an unrealistic mean value compared to the real one; the phase composition in the micro cut-out can show a large discrepancy to the $3 \mathrm{D}$ (real) composition. The major reason for the aforementioned problems is the limited measured area or volume, e.g., as presented in Schneider et al. [7]. The problems, leading to less representative microstructures, exist in both 2D and 3D microstructure cut-outs. If a non-representative microstructure is applied in an FE simulation, the reliability of numerical results would be questionable. For numerical investigations, usually, experimental measurements are already done. Even though there are possibilities for new measurements, the new microstructural cut-outs may still have the same problem. Propagations of microscopic defects and damages very often cause macroscopic failure. Analysis of local hot spots, like zones with stress, strain, or energy concentration, plays an crucial role to improve structural safety and material property, as well as new material design. Hot spot regions have the densest population of mini pores and cracks, part of which quite possibly develop into main cracks. Mechanisms, e.g., the collapse of voids or pores, localized adiabatic shear, dislocation pile-up avalanches, and friction, are possible sources of hot spots. Based on an image of the underlying microstructure, Donegan et al. [8] predicted hot spots through the application of a convolutional neural network method (a sub-branch in machine learning), where the elastic response is modeled using a spectral technique based on fast Fourier transforms. With segmented 3D image of a large size microtomography at hand, the work of Willot et al. [9] reported a combined analytical and numerical study of the extreme values of the thermal and elastic fields occurring in a propellant composite material and put emphasis on the identification of hot-spot zones. Lera et al. [10] determined micro hot spot locations in ceramic superconductors through detected inhomogeneous heating. Consecutively, studies on microstructure were performed to determine defects responsible for hot spot generation. Rimoli et al. [11]. proposed a multi-scale model explicitly accounting for three scales to assess the feasibility of a defect-free initiation mechanism for high energetic materials at the sub-grain scale. They studied shock-induced sub-grain microstructures as possible homogenous sources of hot spots and initiation sites. Reference [12] used deep learning (a sub-field of machine learning) to generate synthetic microstructure for design of novel microstructures of engineer heterogeneous energetic materials. The sensitivity of such materials critically depends on their microstructure and hot spots due to energy localization at sites of porosities and other defects. The precondition of this study is to learn micromechanical features from 
images of real microstructures. In short, it is quite beneficial to use real microstructures to detect hot spots and only a well representative microstructure would lead to trustable results. It is significant to have a way to improve the representativeness of the given microstructure cut-outs.

In order to improve the representativeness of a microstructural cut-out, the current work presents a numerical method to achieve the desired phase volume ratio. If a local morphology possesses a higher volume fraction for the constituents (particles) than the real value, the growth of the matrix is suppressed by their neighbors (constituents around them), i.e., matrix "undergrowth". For the other way around case, the matrix is overgrown. Each local morphology presents a local material status. The current work concentrates on the phase volume ratio and takes a local morphology with a real phase composition as an optimum status resulted from the grain growth. The aforementioned "undergrown" or "overgrown" matrix leads to a local structure presented at a "non-optimum" material status, since the representativeness of the microstructure is not optimal in the sense of phase volume ratio. The real microstructures are given in the form of images based on pixels, which means our method must also deal with pixels. It implies that a minimum area, which can be handled, is identical to the area of one pixel. Here, only square-pixels are considered. As a practical application of this method named as "boundary pixel color alteration (BPCA)", it is to modify the boundary pixel color of grains/clusters/particles. In spite of the pixel color alteration, the original morphological pattern is maintained. As examples, the desired compositions are achieved for four real 2D microstructure cutouts of different materials including polycrystalline MMCs. Our method is also suitable to modify the grain/cluster size or size distribution, since the grain/cluster sizes are changed through the BPCA. 2D microstructures can be applied in 2D, as well as in axisymmetric (2.5D) FE analyses (ABAQUS [13]). This work will show that the phase volume fractions (2.5D axisymmetric analyses), as a function of the structure position to the symmetric axis, converges to the phase area fractions in the $2 \mathrm{D}$ microstructure. A mathematical proof makes the condition clear for this convergence, i.e., this convergence is a general conclusion and implies that it provides a consideration point for all the users encountering the same situation. An improvement of the predicted FE result is presented with the application of a modified microstructure compared to the original one.

\section{Match of Desired Composition}

The current work became true during our numerical investigation of the deformation behavior of polycrystalline $\mathrm{Ag} / \mathrm{SnO}_{2}$ oxide dispersion strengthened (ODS) MMCs. Experimental results can be referred to Wasserbäch et al. [14,15]. For FE simulations, micromechanical features need to be considered, like the local interaction among Ag-Ag grains and $\mathrm{Ag}-\mathrm{SnO}_{2}$ (grain-particle), the Ag grain orientation, the particle size and distribution, as well as the microstructure representativeness. It is well known that the particle phase volume fraction on the microscale is very influential to the homogenized macroscopic $\sigma-\varepsilon$ behavior. If such an FE simulation should predict the deformation behavior in detail, the volume fraction of the strengthening phase should be firstly guaranteed, i.e., the particle volume fraction in the simulation should be approximately the same as the real one.

To reach the real composition, the first consideration was to find a suitable position of the microstructure in the macro cross section (axisymmetric/2.5D analysis), since the element volume is position dependent. Section 2.1 gives the numerical searching result of the phase volume fraction with a dependence on the microstructure position, where a mathematical proof is presented in Appendix A for the numerical result. The crystal plasticity theory used in FE simulations is also briefly presented. Section 2.2 presents a numerical method to solve the mismatch of phase volume/area ratios between the $2 \mathrm{D}$ cut-out and the 3D/real composition. To ensure the confidence of the numerical finding and to test the code, Section 2.3 illustrates results of the volume searching and the pixel modification for three further materials. 


\subsection{Insensibility of Phase Volume Ratios to Microstructure Position in Axisymmetric FE Analyses}

Besides 2D and 3D FE simulations (ABAQUS), the axisymmetry (2.5D) is also an analysis type. To be clear, the real structure geometry in an axisymmetric simulation is not necessarily axisymmetric. Another point is that the coordinate system in an axisymmetric (2.5D) analysis can be the same as the one in a 3D analysis. The geometrical assumption in a 2D analysis is infinite in the third direction. In an axisymmetric analysis, this assumption is finite (Figure 1). Concerning this geometric assumption in the third direction, the axisymmetric analysis (2.5D) has an improvement compared to the 2D one. Micromechanical axisymmetric (2.5D) simulations possess several advantages: (I) such " $2.5 \mathrm{D}$ " simulations are as time efficient as 2D simulations. (II) A full presentation of a variable in all the three directions can be realized in an axisymmetric (2.5D) simulation, but not in a 2D simulation. (III) Especially, it is useful for simulations with a user sub-routine "UMAT" (ABAQUS), since only one "UMAT" is enough for both the axisymmetric (2.5D) and the 3D simulations. Generally, a "UMAT" developed for the 3D case cannot be applied in 2D simulations. (IV) Furthermore, a meshed 2D structure can be directly used in an axisymmetric simulation by simply changing element types into "CAX" element type (ABAQUS). Even though 3D FE analyses generally yield better predicted results than $2 \mathrm{D}$ and axisymmetric ones, 3D simulations are often limited by the calculation capacity, since the total number of elements is easily exceeding the FE calculation limit. For a 3D geometrical adaptive meshing, the phase volume fraction calculated from elements can show a large discrepancy compared to the one calculated by pixels. Still, no concrete relation exists between the phase volume fraction (from elements) after meshing and the one before meshing (from pixels), i.e., this problem is not easy to be under user-control. This volume deviation becomes more serious for fine particle strengthened materials and the difference can be around 10\% and even higher. Comparatively, from our experience, the aforementioned discrepancy is less than $1 \%$ for 2D (axisymmetry) analyses. Besides the analysis types, there are other important factors influencing the accuracy of FE results, like used theories, boundary conditions (BCs) and whether statistical data is large enough. Considering advantages and disadvantages of the three analyzing types, no one can completely replace another, i.e., further coexistence.
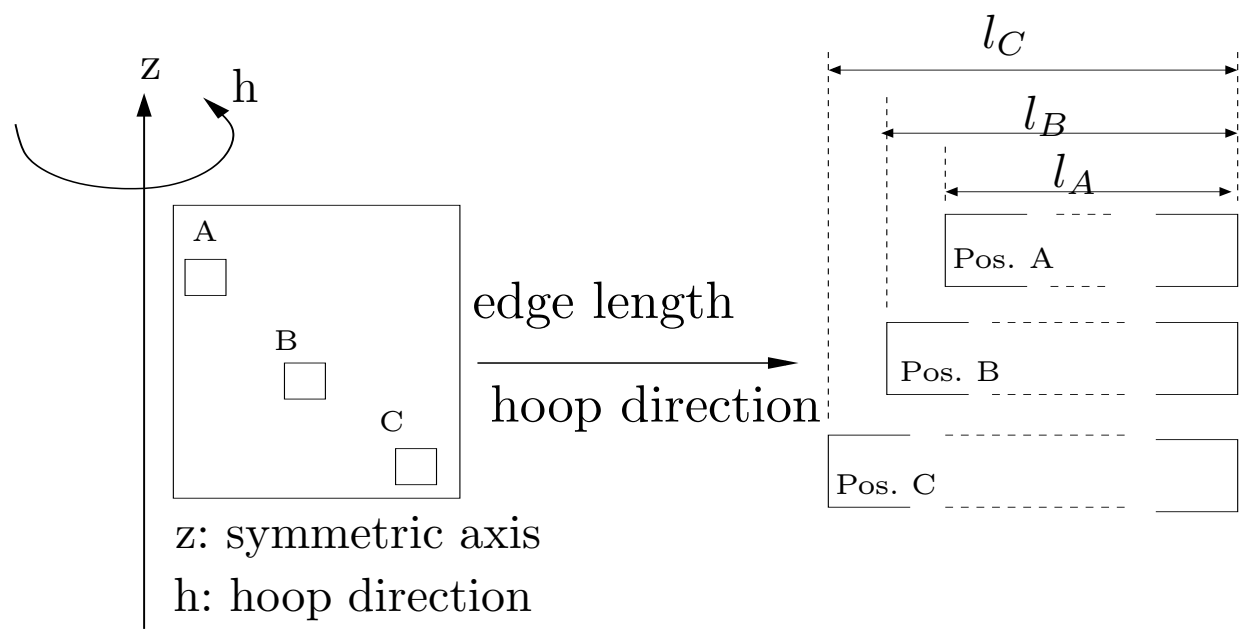

Figure 1. A schematic illustration of different edge lengths in the 3rd direction (hoop direction) for an element at position A, B, and C (Pos. A, Pos. B, and Pos. C) in an axisymmetric cross section (ABAQUS), where the area of this element remains constant.

To investigate the deformation behavior and the texture evolution of the $\mathrm{Ag} / \mathrm{SnO}_{2}$ ODS, the elasto-visco-plastic material model, including the crystal plasticity theory, is applied for polycrystalline Ag grains on the microscopic level. This is realized for the simulation in the form of the user sub-routine "UMAT". The user sub-routine was developed at the Institute of Mechanics of Otto-von-Guericke-University Magdeburg, Germany. In the 
past, it was already applied for various materials under different loading cases [7,16-25]. Section 2.1.1 presents a short description of the applied material model.

For our axisymmetric (2.5D) two-scale FE simulations, the $\mathrm{Ag}: \mathrm{SnO}_{2}$ volume ratio in the microstructure should be the same as the real one $\left(\mathrm{Ag}: \mathrm{SnO}_{2}=83\right.$ vol. $\%: 17$ vol.\%). Here, the "same" ratio means within a tolerable difference, e.g., within 1-2 vol.\%. Our applied microstructures are obtained from EBSD tests. Such images have an original resolution of $1080^{2}$ pixels and dimensions of $80^{2} \mu \mathrm{m}^{2}$. The particle phase is presented in black, e.g., Figure 2a. The $\mathrm{SnO}_{2}$ phase is 24 vol.\% (24\% area fraction) in Figure 2a. In the current work, "vol.\%" symbol is also valid for the area fraction in 2D cases, since it is convenient to compare 3D phase volume ratios with $2 \mathrm{D}$ phase area ratios. The hard particle volume fraction is $7 \mathrm{vol} \%$ higher in the microstructure than in the real one. It is outside of the tolerable range. In order to obtain the real composition, the first step (method) is to find a suitable position for the microstructure in the macro cross section. This method is called "numerical volume searching" in the current work, i.e., finding a suitable position for the microstructure in the macro cross section so that a desired phase volume ratio can be reached. Since the element volume ("EVOL" in ABAQUS) is position dependent. Figure 1 schematically presents this position dependence of the element volume. To be more precise, the volume is position dependent in " $r$ " direction (Figure $3 b$ ), if the area of an element ("CAX" element type) is fixed. The numerical search for the aforementioned suitable position is done in 3 steps: (I) the picture handling based on pixels; (II) the selection of a desired size, and (III) the numerical calculation of phase volume ratios. More in detail:

- (I) The pixel handling is done for a real microstructure of the $\mathrm{Ag} / 17 \mathrm{vol} . \% \mathrm{SnO}_{2}$ ODS (Figure 2a). Figure $2 \mathrm{~b}$ is the resulting image. In Figure $2 \mathrm{~b}$, there are 171 colored $\mathrm{Ag}$ grains, and the $\mathrm{SnO}_{2}$ phase is given in black. For simplicity, the American standard code for information interchange (ASCII) format of images in a grey scale (Figure 2c) is preferred in further steps, since data in this format are convenient for illustration and data processing. In Figure $2 c$, the color of each pixel is described by only one integer in the range of $[0,255]$, i.e., one channel.

- (II) As an example, a small cut-out of the microstructure shown in the upper left corner of Figure $2 b$ (also see Figure $3 a$ ) is selected in search of a desired position, at which the phase volume ratio is $\mathrm{Ag}: \mathrm{SnO}_{2}=83$ vol. $\%: 17$ vol. $\%$. This small cut-out corresponds to about $25^{2} \mu \mathrm{m}^{2}$ and has about $25.5 \%$ area fraction of the $\mathrm{SnO}_{2}$ phase. There are totally $11 \mathrm{Ag}$ grains.

- (III) A code is developed to find a suitable position for the microstructure in the macro cross section. At this suitable position, the volume fraction of the inclusion phase should approximately match the real one. In our example, it is $17 \mathrm{vol} \%$ for the $\mathrm{SnO}_{2}$ phase.

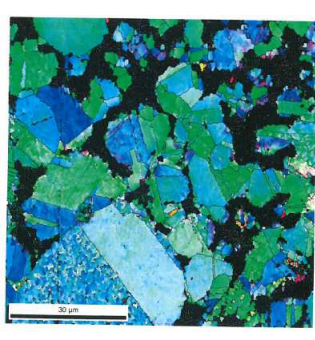

(a)

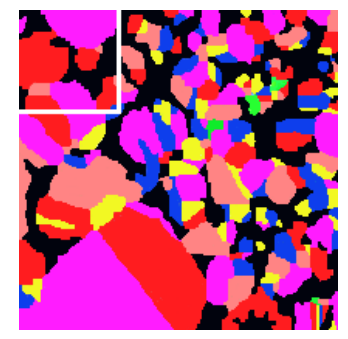

(b)

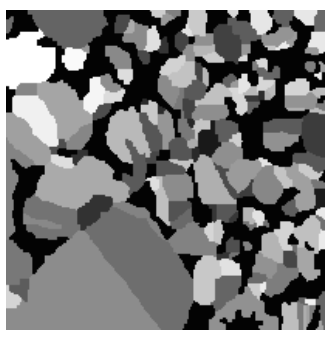

(c)

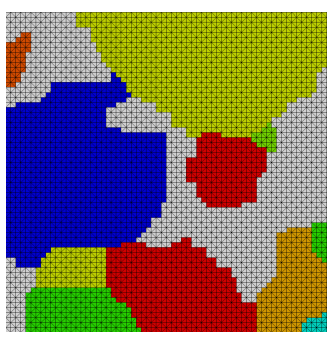

(d)

Figure 2. (a) EBSD image of an $\mathrm{Ag} / 17$ vol. $\% \mathrm{SnO}_{2}$ ODS composite (green body, about 24 vol. $\% \mathrm{SnO}_{2}$ phase in the image); (b) ASCII format picture after pixel handling, where the upper left cut-out (about $25^{2} \mu^{2}{ }^{2}, \mathrm{SnO}_{2}$ phase: $25.5 \%$ area fraction) is applied as the microstructure in Figures 3 and 4; (c) the gray scale image of $(\mathbf{b})$; (d) regular meshing of the microstructure cut-out in the upper left corner of (b). 


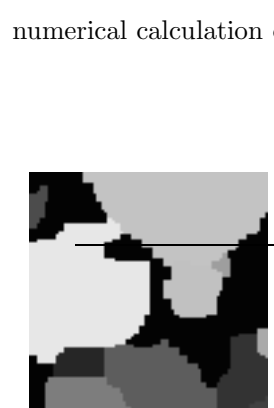

(a)

microstructure length $\mathrm{L}$ microscopic particle vol. \%:
converges to $2 \mathrm{D}$ area\% $\% 25.5 \%$

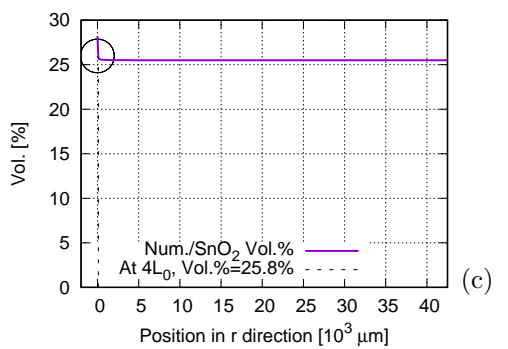

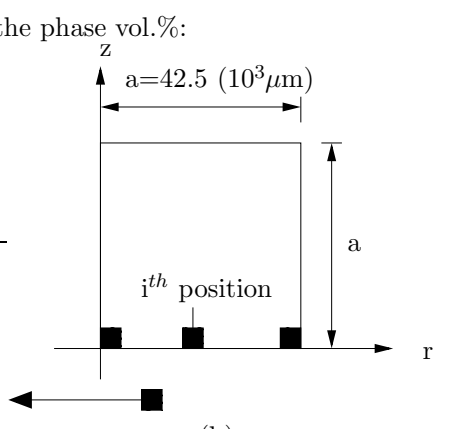

(b)

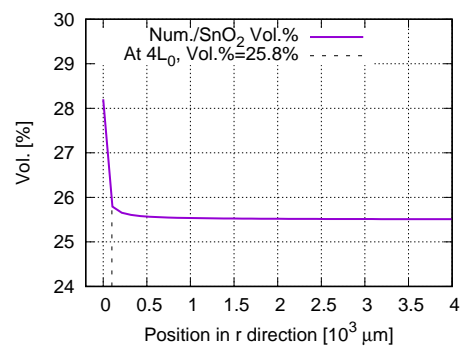

(d)

Figure 3. The numerical calculation of the phase volume fraction of a microstructure: (a) a selected microstructure (upper left corner in Figure $2 \mathrm{~b}$, width $=$ length $=L_{0} \approx 25 \mu \mathrm{m}, \mathrm{SnO}_{2}$ phase area fraction $\approx 25.5 \%$; (b) a schematic illustration of the microstructure position in a macroscopic cross section (axisymmetry/2.5D in ABAQUS), where the whole microstructure is presented by a black square; (c) the $\mathrm{SnO}_{2}$ phase volume fraction in an axisymmetry (2.5D) simulation converges to its area fraction in the $2 \mathrm{D}$ microstructure, i.e., the indifference of the phase volume fraction to the microstructure position; (d) an enlarged view of the region marked by a circle in (c).

Our samples have a diameter of $5 \mathrm{~mm}$ after two passes of hot extrusions. The ones before the extrusion have a diameter of $85 \mathrm{~mm}$, i.e., the chamber for the extrusion has a diameter of $85 \mathrm{~mm}$. Figure 2a corresponds to a material status before extrusion. It means that Figure $2 \mathrm{a}$ is a cut-out from a green body. Figure $3 \mathrm{a}$ is the aforementioned selected microstructure cut-out for the numerical volume searching process. Figure $3 \mathrm{~b}$ schematically presents the macroscopic cross section of a green body sample with a radius of $42.5 \mathrm{~mm}$. In Figure $3 b$, " $z$ " and " $r$ " show the axisymmetric and the transverse direction, respectively. The black square presents the whole microstructure, i.e., Figure 3a. During the search for a position for the microstructure with $17 \mathrm{vol} . \% \mathrm{SnO}_{2}$, the step length in " $r$ " direction is $0.0741 \mu \mathrm{m}$. This step length corresponds to the length presented by one pixel in our original EBSD image $\left(\frac{80 \mu \mathrm{m}}{1080 \text { pixels }} \approx 0.0741 \mu \mathrm{m}\right.$ per pixel). Figure $3 \mathrm{c}$ presents the numerical volume searching result. From Figure $3 c$, it is clear that the $\mathrm{SnO}_{2}$ phase volume fraction is indifferent to the position of the microstructure in the macrostructure. The phase volume fraction converges to its area fraction in the 2D microstructure (25.5 vol.\%). Figure $3 \mathrm{~d}$ shows an enlarged view of the region marked by a circle in Figure $3 c$. This convergence means that it is not possible to find a position for the given microstructure with 17 vol.\% $\mathrm{SnO}_{2}$. Figure $3 \mathrm{c}$ is the result from our numerical searching, it would be more convincing, when phase volumes in FE simulations show the same independence on the microstructure position. Since two-scale prediction is preferred in our work, the aforementioned indifference is also proved by a two-scale FE meshing. Figure 4a illustrates the dimensions of the figures in Figure $4 \mathrm{~b}$. The same microstructure cut-out (Figure 3a) is embedded in four different positions inside a macrostructure as shown in Figure $4 \mathrm{~b}$, left. The meshing of the transition zone is shown in Figure $4 \mathrm{~b} /$ middle for connecting positions with the macrostructure. For the connecting positions with the microstructure, the meshing of the transition zone is presented in Figure $4 b$, right. Each pixel in the microstructure is meshed 
with 2 triangles (Figure $4 \mathrm{~b}$, right) so that the volume covered by a pixel is identical for the numerical search (Figure 3 ) and for the FE simulation (at a specific position of the microstructure on the macro cross section). The resulting volume fractions of the $\mathrm{SnO}_{2}$ phase and $11 \mathrm{Ag}$ grains are shown in Figure 4c. For the $\mathrm{SnO}_{2}$ phase, its volume fraction is marked by a black circle. It is pointed out that the phase volume fraction indifference to the microstructure position is valid for any phase and any microstructure size in an axisymmetric (2.5D) analysis. To prove the universality of this convergence, a mathematic proof is presented in Appendix A.

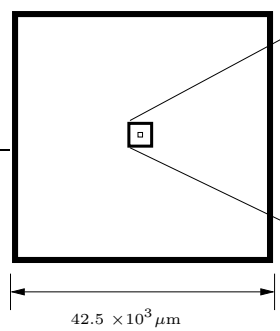

$42.5 \times 10^{3} \mu \mathrm{m}$

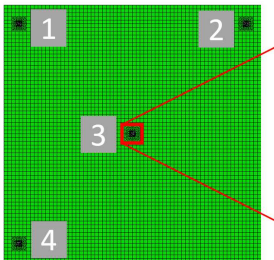

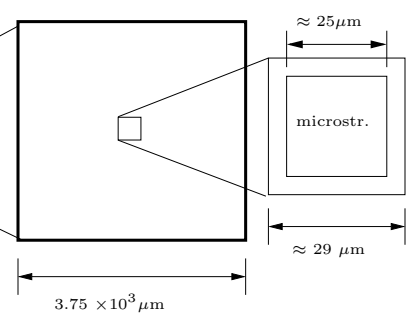

(a)

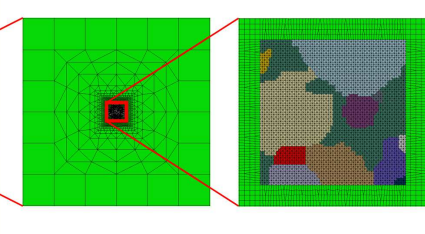

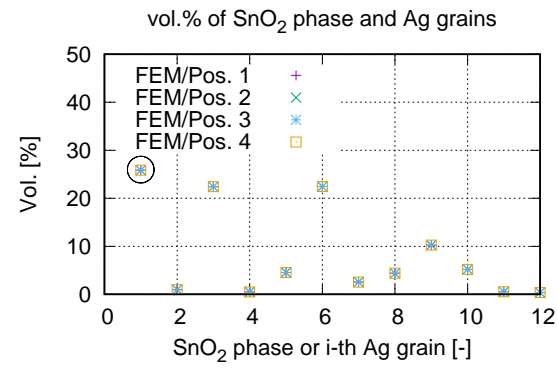

(c)

(b)

Figure 4. The FE simulation proof of the phase volume fraction indifference to the microstructure position: (a) dimensions of the macrostructure, the cut-outs and the microstructure; (b) four different positions of the microstructure cut-outs (Figure 3a) to show the meshing; (c) the indifference of the volume fraction of the $\mathrm{SnO}_{2}$ phase (marked with a circle) and $11 \mathrm{Ag}$ grains to their position in the macrostructure.

\subsubsection{Crystal Plasticity Modeling}

For a good numerical prediction of the polycrystalline material, like our ODS Ag/ $\mathrm{SnO}_{2}$ MMCs, different micro mechanisms should be taken into consideration, like the anisotropy, the interaction among grains, and the dislocation activation, as well as the heterogeneity. Large plastic deformation on the microlevel requires a material model, which can describe the local distorsion and dilatation well. The elasto-visco-plastic material model from the crystal plasticity is suitable to mechanically simulate the Ag phase deformation behavior on the microscopic level. To realize FE simulations, a user defined sub-routine (UMAT in ABAQUS) was developed at the Institute of Mechanics (IFME Institute) of Otto-vonGuericke-University Magdeburg, Germany $[7,16,26,27]$. To be simple, the applied model is briefly described as follows.

Elastic Law

A finite anisotropic linear elastic law is used (Equation (1)), in which the 2nd PiolarKirchhoff stress tensor $T^{2 P K}$ is a function of the Green strain tensor $E$ :

$$
\boldsymbol{T}^{2 P K}=\mathbb{K}[\boldsymbol{E}] \text { with } \boldsymbol{E}=\frac{1}{2}\left(\boldsymbol{F}^{\top} \boldsymbol{F}-\boldsymbol{I}\right), \boldsymbol{F}=\boldsymbol{F}_{e} \boldsymbol{P}^{-1}=\boldsymbol{F}_{e} \boldsymbol{F}_{p} .
$$

In Equation (1), $\mathbb{K}$ and $I$ represent the 4 th order elasticity tensor and the identity tensor, respectively. $\boldsymbol{F}$ denotes the deformation gradient tensor, which can be further multiplicatively decomposed into $\boldsymbol{F}_{e}$ and $\boldsymbol{P}$, where $\boldsymbol{P}$ indicates the plastic transformation [28,29], and $\boldsymbol{F}_{e}$ is defined as $\boldsymbol{F}_{e}=\boldsymbol{F} \boldsymbol{P}$. If $\boldsymbol{P}^{-1}=\boldsymbol{F}_{p}$ is identified, this $\left(\boldsymbol{F}=\boldsymbol{F}_{e} \boldsymbol{P}^{-1}\right)$ leads to the same decomposition suggested by Lee (1969) [30], i.e., $\boldsymbol{F}=\boldsymbol{F}_{e} \boldsymbol{F}_{p}$. This elastic law 
(Equation (1)) is not constant in time after yielding. It is convenient to transform it into a time-independent law:

$$
\boldsymbol{T}_{e}^{2 P K}=\tilde{\mathbb{K}}\left[\boldsymbol{E}_{e}\right] \quad \text { with } \quad \boldsymbol{E}_{e}=\frac{1}{2}\left(\boldsymbol{P}^{\top} \boldsymbol{F}^{\top} \boldsymbol{F} \boldsymbol{P}-\boldsymbol{I}\right)=\frac{1}{2}\left(\boldsymbol{F}_{e}^{\top} \boldsymbol{F}_{e}-\boldsymbol{I}\right) .
$$

Flow Rule

The flow rule is taken from the finite crystal visco-plasticity theory, in which the time evolution of $\boldsymbol{P}$ is specified in terms of the shear rate $\dot{\gamma}_{\alpha}$ and the Schmid tensors $\boldsymbol{M}^{\alpha}$ with a certain slip system $\alpha . \dot{\gamma}_{\alpha}$, the Schmid resolved shear stress $\tau^{\alpha}$ and $\boldsymbol{M}^{\alpha}$ are given as:

$$
\dot{\gamma}_{\alpha}=\dot{\gamma}_{0} \operatorname{sgn}\left(\tau_{\alpha}\right)\left|\frac{\tau_{\alpha}}{\tau_{\alpha}^{\alpha}}\right|^{m}, \quad \tau_{\alpha}=\tilde{\mathbb{K}} T_{e}^{2 P K} \cdot \tilde{\boldsymbol{M}}^{\alpha} \approx T_{e}^{2 P K} \cdot \tilde{\boldsymbol{M}}^{\alpha}, \quad \tilde{\boldsymbol{M}}^{\alpha}=\tilde{\boldsymbol{d}}_{\alpha} \otimes \tilde{\boldsymbol{n}}^{\alpha},
$$

respectively [31]. $\dot{\gamma}_{0}$ and $\tau_{\alpha}^{C}$ correspond to the reference shear rate and the critical resolved shear stress. For a given spatial velocity gradient $L:=\dot{\boldsymbol{F}} \boldsymbol{F}^{-1}$, the flow rule can be formulated in terms of $\boldsymbol{F}_{e}$,

$$
\dot{\boldsymbol{F}}_{e} \boldsymbol{F}_{e}^{-1}=\boldsymbol{L}-\boldsymbol{F}_{e} \tilde{\boldsymbol{k}}\left(\boldsymbol{T}_{e}^{\prime}, \tau_{\alpha}^{C}\right) \boldsymbol{F}_{e}^{-1}, \quad \tilde{\boldsymbol{k}}\left(\boldsymbol{T}_{e}^{\prime}, \tau_{\alpha}^{C}\right)=: \sum_{\alpha=1}^{N} \dot{\gamma}_{\alpha}\left(\boldsymbol{T}_{e}^{\prime}, \tau_{\alpha}^{C}\right) \tilde{\boldsymbol{M}}^{\alpha},
$$

with the Mandel stress tensor $\boldsymbol{T}_{e}=\boldsymbol{F}_{e}^{\top} \boldsymbol{T}_{e}^{K} \boldsymbol{F}_{e}^{-\mathrm{T}}$.

\section{Hardening Rule}

The Kocks-Mecking hardening rule [32,33] is applied, which is a type of the Voce rule and emphasizes the mechanisms of the dislocation density growth, the accumulation and the density decrement. The ansatz applied here has the form

$$
\dot{\tau}^{C}=\Theta_{0}\left(1-\frac{\tau^{C}}{\tau_{c}^{v}\left(\tau_{\alpha}, \tau^{C}\right)}\right) \dot{\gamma}\left(\tau_{\alpha}, \tau^{C}\right), \quad \text { where } \quad \tau_{c}^{v}=\tau_{c 0}^{v}\left|\frac{\dot{\gamma}\left(\tau_{\alpha}, \tau^{C}\right)}{\dot{\gamma}_{0}^{*}}\right|^{\frac{1}{n}} .
$$

$\Theta_{0}$ is the work hardening rate near to the yielding point. $\tau_{c 0}^{v}$ is an input material parameter and can be identified from the experiment [33]. $\dot{\gamma}_{0}^{*}$ is a material constant $\left(10^{7} \mathrm{~s}^{-1}\right)$. Other values of $\dot{\gamma}_{0}^{*}$ are possible, but it should be kept in agreement with the order of magnitude expected from the dislocation theory [33]. The reference shear rate $\dot{\gamma}_{0}$ is a constant.

\section{Homogenization}

RVE is used for the microstructure in the current work in order to achieve the transition from the micro to macro variables. The macroscopic material variables are obtained through the homogenization of the corresponding micro fields. Based on the postulate of the work equivalence on the micro and the macro scale [34], the global first Piola-Kirchhoff stress $\left(\bar{T}^{1 P K}\right)$ is related to the local one $\left(T^{1 P K}\right)$ by

$$
\overline{\boldsymbol{T}}^{1 P K}=\frac{1}{V} \int_{V} T^{1 P K} \mathrm{~d} V .
$$

\subsection{A Numerical Method to Match Real Composition for Microstructure Cut-Outs}

Concerning the physical background of our method to modify the microstructure, it can be referred to Appendix B. The precondition for the application of our method is that the representativeness of the microstructure is already fulfilled to a certain extent. Here, the aim is to improve the representativeness and put emphasis on the phase volume ratio matching the reality exactly. To achieve a desired volume fraction for a given phase, the basic idea is the alteration of the boundary pixel color of this phase, i.e., our method BPCA. When the volume fraction of the considered phase is larger than the real composition, the color of its outer boundary pixels will be changed into its neighboring pixels' colors. This method is also suitable for the-other-way-around case, i.e., when the volume fraction of a considered phase in a cut-out is smaller than the value in the real composition. In this case, colors 
of some neighboring pixels would be changed to the pixel color of this considered phase. The ASCII format is preferred for this color changing process. A code was developed to execute this modification process.

In the current work, each pixel is square shaped, i.e., a pixel presents the same absolute dimension in both horizontal and vertical directions. Figure 5 a schematically shows a considered pixel (in black square) with its neighbors in an image in the ASCII format. The region DEFG in Figure 5a presents a microstructure. The point E coincides with the coordinate origin. In Figure $5 \mathrm{a}$, the pixel shown in a black square is the current considered pixel. For pixel types A, B, and C in Figure 5a, the number of total neighboring pixels is used as the categorizing condition. In our example, the $\mathrm{SnO}_{2}$ particle phase has a higher volume fraction than the real one. The color alteration will happen only to the outer boundary pixels of the $\mathrm{SnO}_{2}$-phase. Such pixels have neighbors which present $\mathrm{Ag}$ grains. If neighboring pixels have more than one different colors, a priority criterion should be given to determine the new color for the considered pixel. Figure $5 \mathrm{~b}$ demonstrates one of the possibilities. In Figure $5 b$, the smaller numbers have priority compared to larger numbers. Supposing that the particle phase has a smaller volume fraction than the real one, the pixel color assignment would be easier than in the aforementioned case. The neighboring pixels (Ag grain pixels) would just change into the color black. No priority for the color assignment is required.

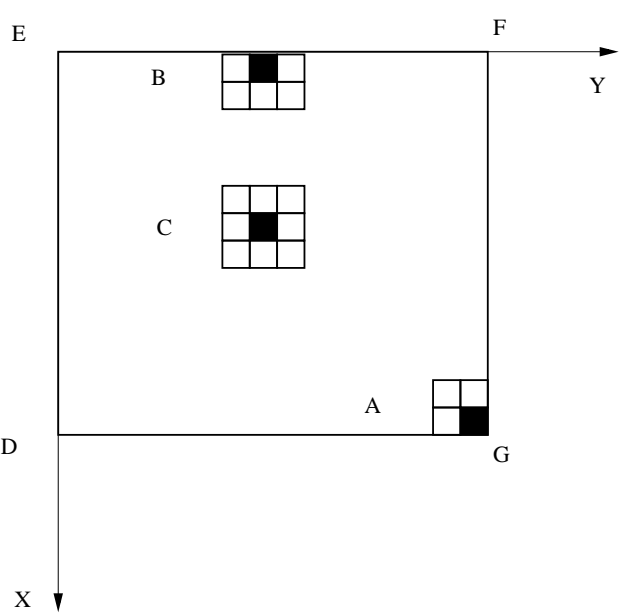

(a)
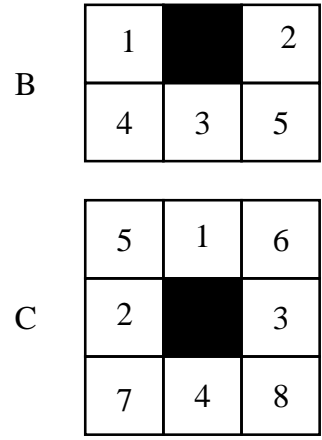

A

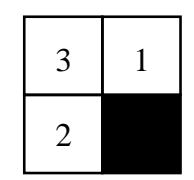

(b)

Figure 5. (a) A sketch of a pixel image to illustrate the 3 types of pixels (square in black) with different numbers of total neighbors (white squares); (b) put the neighbors in order: a small number has the privilege compared to a larger number for the color assignment.

It is supposed that a $2 \mathrm{D}$ image has $N_{x}$ and $N_{y}$ pixels in the $\mathrm{X}$ and $\mathrm{Y}$ direction, respectively (for directions, see Figure 5a). $N_{x}$ and $N_{y}$ present integers. The number of total pixels will be $N=N_{x} \times N_{y}$. The pixel position can be identified as $P_{i}(x, y)$ with $x \in\left[1, N_{x}\right]$, $y \in\left[1, N_{y}\right]$ and $i \in[1, N]$. Each pixel with a square shape has four peak points. They can be presented as four nodes with possible coordinates of $P_{i}^{1}(x, y)$ to $P_{i}^{4}(x, y)$. The $x$ and $y$ values in $P_{i}^{1}(x, y)$ to $P_{i}^{4}(x, y)$ have ranges $x \in\left[0, N_{x}+1\right]$ and $y \in\left[0, N_{y}+1\right]$ in the $\mathrm{X}$ and $Y$ direction, respectively. To reach the real material composition, Figure 6 describes this modification process in 5 steps $(\mathrm{I}-\mathrm{V})$ :

- $\quad$ For step-I, the free software GIMP with an open access is used. The given example (Figure 2c) possesses $171 \mathrm{Ag}$ grains. It means that 172 color numbers (172 pixel groups) are enough to identify every $\mathrm{Ag}$ grain and the $\mathrm{SnO}_{2}$ phase for step-I. For a more complex structure with more than 256 grains/clusters, our method is still applicable. 
The method described in step-II-b can be used to separate grains/clusters with the same pixel color number.

- In step-II, all the information concerning a pixel should be coupled (linked with each other). It includes the pixel position $\left(P_{i}(x, y)\right)$, the pixel group number, four node coordinates $\left(P_{i}^{1}(x, y)\right.$ to $\left.P_{i}^{4}(x, y)\right)$ and the color number. The number of total pixel groups is identical to the number of total pixel colors at this stage. It means that pixels with the same color are in the same group, even though they may belong to different pixel groups later on. Step-II-a is automatically done by reading the input data, since all the particles have the same color. Step-II-b is a key point for the pixel color alteration. A "glue" process is iteratively applied here. As the starting point, the code takes an arbitrary pixel (pixel-a) in the considered range. This range means a considered phase or grain. The aforementioned starting pixel belongs to a certain cluster A. It means that different clusters will be identified from the particle phase. Each cluster starts with a pixel. The cluster A is also the pixel group A. If another pixel fulfills two conditions: (i) being inside the considered range (black color in the example); (ii) having at least one shared node $\left(P_{i}^{1}(x, y)\right.$ to $\left.P_{i}^{4}(x, y)\right)$ with the pixels inside the pixel group-A (already identified), this pixel will be included to the pixel group A. Thus, the number of total pixels in group A increases. Then, the code will search for the next possible pixel. During the search, there are parallel sub-groups for a pixel group, since the iterative loop is done for pixels $P_{i}(x, y)$ one after another. It begins with $x=1$ and $y=1$, and goes to $x=1$ and $y=2$. After the pixel $P\left(x=1, y=N_{y}\right), P(x=2, y=1)$ will be the next pixel to be considered. It implies that this "glue" process includes: (a) sticking pixels to pixels; (b) sticking sub-groups to sub-groups; (c) sticking pixels and sub-groups. As a final result of step-II-b, individual particle clusters are identified.

- Step-III links together the information attached to a pixel. It can be relatively easily done compared to the step-II. For each identified particle cluster, a new pixel color is required to distinguish it from other clusters and Ag grains. When it requires more than 256 grains/clusters in a microstructure, some grains/clusters will have the identical pixel color number. In this case, in order to track each grain/cluster, one can resort to the integer assigned by the code (mentioned in Step-I). No neighboring grains/clusters share the same pixel color number. The considered field is the microstructural features of alloys. The possibility should be nearly zero for one grain/cluster with more than 255 neighbors. Another way would be to use the colored pixels. For such pixels, 3 numbers (red-green-blue RGB color) describe one pixel color. But the work of the pixel color comparison for the code will be much higher than the comparison work used by the greyscale color.

- Step-IV identifies the outer boundary pixels of an individual pixel group inside the considered range. In our case, this range refers to $\mathrm{SnO}_{2}$ clusters. This step is another key point of the algorithm. The criterion is that a $\mathrm{SnO}_{2}$ pixel has at least one neighbor with a pixel color number of Ag grains. As a verification, all the pixels on the outer boundary must have at least one shared node with one of the other pixels in the same pixel group (the boundary pixel group, not the cluster pixel group). Each boundary pixel group composes a closed loop which encloses the given grain/cluster. Such closed loops can be referred to, e.g., the particle surrounded by a green oval line in Figure $7 \mathrm{~b}, \mathrm{e}$ (for particles inside the cut-out, not the ones cut by the microstructure edges). It is pointed out that some $\mathrm{SnO}_{2}$ boundary pixels are not shown in Figure $7 \mathrm{~b}, \mathrm{c}, \mathrm{e}$. Such pixels locate on the edge of the microstructure cut-out. By adding such pixels in Figure $7 \mathrm{~b}, \mathrm{c}, \mathrm{e}$, closed loops will also be shown for particles cut by the microstructure edges. Such images with closed loops for all the particles would not be presented in the current work, since it is just a matter of choosing the image illustration and has no influence on our method. $\mathrm{SnO}_{2}$ boundary pixels located on microstructure edges do not have Ag neighbors, so they are excluded of the pixel 
color alteration process. It also means that their color is maintained during the pixel color modification process.

- $\quad$ Step-V alters and updates the pixel colors. If the desired volume fraction is not yet reached, the update of the neighborhood information will also be done so that it is ready for the next layer of the BPCA process. As a final result, the real composition is achieved, and the pattern of the original microstructure is maintained, as well.

In our early work [35], where no method description is available due to predefined page limit, a small cut-out with only $11 \mathrm{Ag}$ grains and $3 \mathrm{SnO}_{2}$ clusters (upper left corner in Figure 2b) was used to test the aforementioned BPCA process. By taking Figure 2c $\left(80^{2} \mu \mathrm{m}^{2}\right)$ as the starting microstructure, Figure $7 \mathrm{a}-\mathrm{f}$ show the intermediate and the final results of this modification process. Based on our experience, the grain and particle boundaries are already well recognizable in an image with $200^{2}-300^{2}$ pixels for the aforementioned size $\left(80^{2} \mu \mathrm{m}^{2}\right)$. The particle phase amounts to 23.96 vol.\% in Figure $2 \mathrm{~b}, \mathrm{c}$. Figure $7 \mathrm{a}$ shows the identified particle phase as a whole. It means that all the particle pixels are in the same pixel group at this stage and have the same color black. Figure $7 \mathrm{~b}$ presents the boundaries of 25 identified particles, where each individual one has its own color. Here, only the pixels of the particle phase (on particle boundaries) are shown, which have neighboring pixels belonging to $\mathrm{Ag}$ grains. It is pointed out that some particles may not be well identified by naked eyes in Figure $7 \mathrm{~b}$.

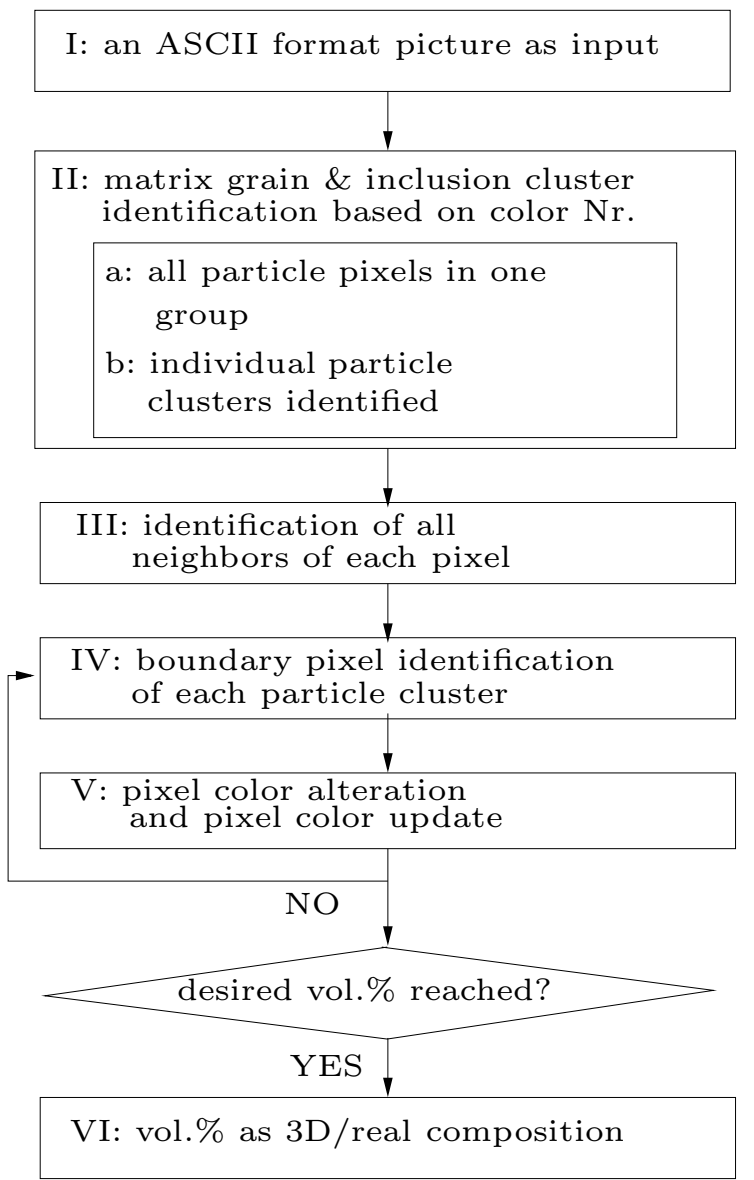

Figure 6. A flow chat to show the process of pixel color alteration of a $2 \mathrm{D}$ microstructure to obtain desired 3D/real composition for a polycrystalline/multiphase material. Here, the vol.\% is just a symbol for the area fraction. 


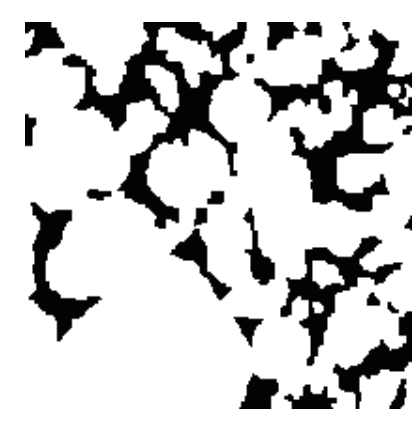

(a)

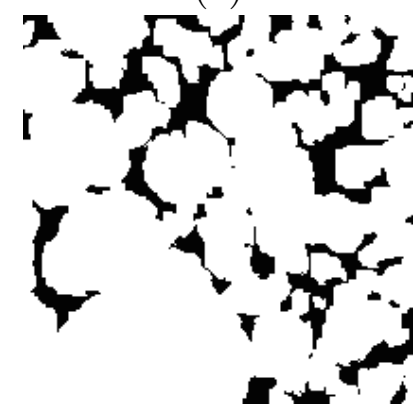

(d)

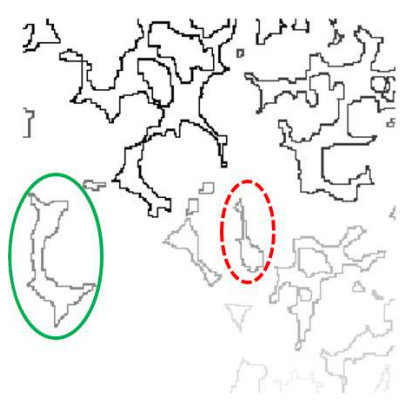

(b)

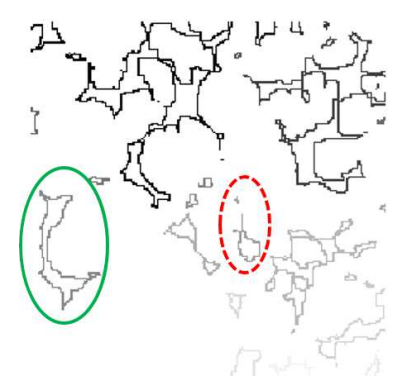

(e)

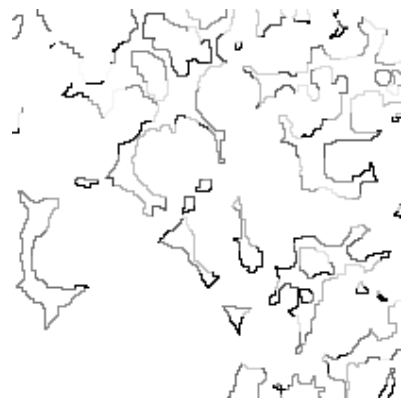

(c)

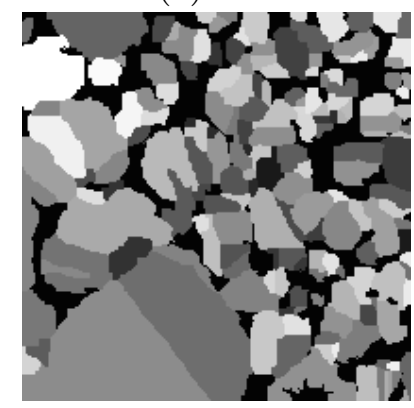

(f)

Figure 7. The boundary pixel color alteration (BPCA) procedure to achieve the real phase composition (the original microstructure: Figure 2c): (a) all identified $\mathrm{SnO}_{2}$ pixels with the same black color (area fraction about 24\%); (b) identified boundary pixels of individual particle clusters (colors artificially assigned for cluster illustration); (c) after the BPCA process (here, pixels partly with Ag properties); (d,e) images corresponding to $(\mathbf{a}, \mathbf{b})$ for the second round of BPCA; (f) final morphology with a real composition and directly ready for meshing.

For a given microstructure, the number of total pixels $P^{\text {all }}$ is fixed. $i$ refers to the number of an individual particle/cluster in a given microstructure. In our case, $i \in$ $[1,25]$ (Figure $7 \mathrm{~b}, \mathrm{c}, \mathrm{e})$. vol ${ }^{i}$ denotes the $i$ th cluster volume/area fraction. $\mathrm{vol}^{\mathrm{SnO}_{2}}$ presents the $\mathrm{SnO}_{2}$ phase volume/area fraction, i.e., $\sum \mathrm{Vol}^{i}=\mathrm{Vol}^{\mathrm{SnO}_{2}}$. For the given example (Figure 7), $\mathrm{Vol}^{\mathrm{SnO}} \mathrm{O}_{2}=23.96 \%$ before the modification (Figure 7a) and $\mathrm{Vol}^{\mathrm{SnO}_{2}}=17 \%$ after the modification (Figure 7f). $\Delta v_{o l} \mathrm{SnO}_{2}$ is the difference of the particle phase volume fraction between the original 2D microstructure cut-out and the 3D/real one, i.e., $\Delta v_{0} l^{S_{n O}}{ }_{2}=$ $23.96 \%-17 \%=6.96 \% . P^{i}$ presents the total pixel number of the ith particle cluster at the beginning of this modification process, and $v o l^{i}=\frac{P^{i}}{\text { Pall }}$. If $P_{m}^{i}$ indicates the number of total modified pixels on the boundary of the cluster $i$, then $\left(P_{m}^{i}<P^{i}\right)$ :

$$
P_{m}^{i}=\frac{v o l^{i}}{v o l^{S n O} O_{2}} \Delta v o l^{S n O_{2}} P^{a l l} .
$$

In our example (Figure 2c), $v \mathrm{ol}^{\mathrm{SnO}_{2}}=23.96 \%, \Delta v_{0} \mathrm{SnO}_{2}=6.96 \%$ and $P^{\text {all }}=42,436=$ $206 \times 206$. The largest particle cluster has 2882 pixels $\left(P^{i}={ }^{\max } P^{i}=2882\right)$. For this particle, it holds $v_{0} l^{i}={ }^{\max }$ vol $^{i}=\frac{2882}{42,436}=0.06791 \%$ and

$$
{ }^{\max } P_{m}^{i}=\frac{0.06791 \%}{23.96 \%} \times 6.96 \% \times 42,436 \approx 837 .
$$

The number of total $\mathrm{SnO}_{2}$ pixels with a color alteration will be $6.96 \% \times 42,436=$ 2801 for the particle phase in our example. It also means that a particle with a smaller volume would have fewer pixels changed into Ag colors. As a result, all particles in the original microstructure are involved in the BPCA process, as well as maintained in the final microstructure (after the pixel color modification). From Figure $7 \mathrm{~b}, \mathrm{c}$, some pixel colors of the $\mathrm{SnO}_{2}$ phase are changed into the color of polycrystalline Ag grains. These Ag grains must include pixels which are neighbors of particle pixels in Figure $7 \mathrm{~b}$. The involved pixels 
in Figure $7 b, c$ are at the same positions. But each grey colored pixel in Figure $7 \mathrm{c}$ has the property of a $\mathrm{Ag}$ grain, not the property of $\mathrm{SnO}_{2}$ anymore. It is worthy to mention that some pixels in Figure 7c might not be identified by the naked eyes, since the color contrast to the white background is too weak. After the pixel color change for the first layer (outer boundaries of particle clusters), the resulted microstructure, not shown here, is similar to Figure 2f, which is an intermediate result, since the $\mathrm{SnO}_{2}$ phase has not yet reached its real volume fraction. The pixel color modification should go further, i.e., to the 2nd layer or $(\mathrm{N}+1)$ th layer in order to reach the desired phase volume fraction. Before going to the next layer, the neighborhood update in the code is necessary for pixels located on particle boundaries, since the boundary pixels of the $\mathrm{SnO}_{2}$ phase are not identical to the ones before the color change. All the neighbors of a pixel on boundaries of clusters need to be identified.

Some clusters of the $\mathrm{SnO}_{2}$ phase might be sub-divided into sub-clusters due to the pixel color changing. If so, the presented code can still identify all the outer boundaries for the whole $\mathrm{SnO}_{2}$ phase, since the iterative process in the code goes through all the pixels in the considered range, i.e., all pixels in color black. The particle marked with a red dashed oval line in Figure $7 \mathrm{~b}$ is separated into two particles (particles marked with a red oval line in Figure 7e) after the pixel color alteration. Analogous to Figure 7a,b, Figure 7d,e illustrate the modification process for the 2 nd layer, the starting state of which is Figure $7 \mathrm{~d}$ for the boundary pixels of the particle phase. The 2 nd round of searching boundary pixels includes only the particles, the $P_{m}^{i}$ of which in Equation (7) is not yet reached. The final result is presented in Figure $7 \mathrm{f}$, which is completely ready for meshing. Based on our experience, the particle volume fraction after meshing, i.e., calculated from elements, has a deviation to the value calculated from pixels less than $1 \%$, which is inside an acceptable tolerance. In our case, the former is slightly lower than the later. In such a case, one tip would be to modify the given cut-out to a particle fraction $0.5 \%$ higher than the real one.

The next work is to apply the resulted image in an axisymmetric $(2.5 \mathrm{D})$ two-scale FE model to simulate the extrusion process. Such FE calculations (phase field method [36]) should predict the recrystallization mechanisms of Ag grains. Special attention will be paid to the formation of $\Sigma$ 3-twins in Ag phase. After twice extruded, the resulting microstructure will be loaded under tension to investigate the $\Sigma 3$-twins influence on the texture evolution. FE results will be given in further reports.

Our BPCA method is also applicable to modify $\mathrm{N}(\mathrm{N}>1)$ phases in a microstructure. Each phase can be polycrystalline. In such a case, one way would be to perform BPCA for phases one after another. During the modification of the $m$ th phase $(1<\mathrm{m} \leq \mathrm{N})$, the pixels, which belong to the already modified pixels of phase $\mathrm{k}(1 \leq \mathrm{k}<\mathrm{m})$, should be preserved, i.e., not taking part in the BPCA procedure. This is to guarantee the correct volume/area fraction for the previously modified phases. Our code does not include this function to preserve pixels, since no such necessity, i.e., no modification of N phases, appears in our works until now. As mentioned in Section 1, our method is also applicable to modify the size or size distribution of a phase or several phases, since it goes through every pixel and the pixel color alteration changes the size of the phase/grain. If a grain/cluster has necking regions (narrow regions between two or more broad regions), the BPCA process can separate it into two or more grains/clusters (e.g., the particle surrounded by a dashed red oval line in Figure $7 \mathrm{~b}, \mathrm{e}$ ). It indicates that the distribution of grains/clusters is modified. It is pointed out that the modification of the phase distribution is not the aim of the current work. Simply, a particle with a necking region is separated by the BPCA process (Figure $7 \mathrm{~b}, \mathrm{e}$ ). The original particle distribution pattern is mostly maintained in our example. As mathematically shown in Appendix A, the phase volume fraction in a microstructure can be indifference to the microstructure's position in an axisymmetric (2.5D) analysis (Figure A1 in Appendix A). Our microstructure modification method is even more meaningful, since the resulting microstructures can be applied in 2D, as well as in axisymmetric (2.5D) FE simulations. 


\subsection{Further Applications}

To show the universality of the numerical finding, i.e., the phase volume fraction convergence in an axisymmetric simulation to its $2 \mathrm{D}$ area fraction, and to train our code, the same progress as given in Figure 7 is applied for another three material microstructures.

As a kind of tool materials, tungsten carbide/cobalt (WC-Co) exhibits properties with exceptional combinations of hardness, toughness, strength and wear resistance. They are important for industrial manufacturing and daily life. Hard metal inserts share half of the market for cutting tools [37]. For applications of hard metal components, such as cutting tools and drawing dies, the cemented WC-Co share about $98 \%$ of the market today [38]. Concerning these WC-Co tool materials, most published results are for the ones with tungsten carbide phase as the major phase (matrix). Some of our studies concentrate on the deformation and the damage evolution in $\mathrm{Co} / \mathrm{WC} /$ diamond MMCs with Co as the matrix. Our sample material, much less studied as compared to those with WC as the matrix, is also a kind of tool materials, e.g., for cutting and grinding. Such materials can show both ductile and quasi-brittle behaviors [39,40]. Figure 8a presents a real 2D microstructure cut-out of a Co/WC/diamond composite with 9.06 vol.\% diamond, where the volume ratio of Co:WC:diamond is 90:5:5 for the commercial sample. After the pixel handling, Figure $8 \mathrm{~b}$ with a pixel resolution of $407 \times 322$ illustrates the same microstructure as given in Figure 8a. Figure 8c shows the identified boundary pixels of the 54 diamond particles, where each individual piece is assigned with a non-equal pixel color number. The diamond phase volume fraction in an axisymmetric analysis is presented according to the microstructure position in the macrostructure, where the length of one pixel is used as a unit length. The vol. $\%$ of the diamond phase with a value of $8.62 \mathrm{vol} . \%$ at the beginning of the calculation and $9.05 \mathrm{vol} . \%$ at the end (Figure 8d) converges to its 2D area fraction of $9.06 \%$ in the microstructure. At the position with $r=4 L_{0}$, i.e., $r=4 \times 407=1628$ unit lengths, the diamond volume fraction is $9.01 \mathrm{vol} \%$. There exist WC particles with a non-negligible volume fraction with a sub-micro-size, which cannot be captured by a microstructure. In this case, the size and strengthening effect of sub-micron sized WC particles are taken into consideration by using the homogenized matrix ( $\mathrm{Co}+$ sub-micron-sized-WC) in the FE simulation, where the mechanism-based strain gradient theory $[41,42]$ is applied in an axisymmetric simulation. The resulted homogenized matrix is further used in 2D FE simulations for the local damage evolution, since only a measured 2D local strain map is available due to limited experimental data. As mentioned in Section 1, the analysis types, i.e., 2D, axisymmetry and 3D, have coexisted and will further coexist. For one-scale micromechanical FE simulations, periodic boundary conditions (PBCs) should lead to better predictions than homogeneous ones, since the latter set too strong constraints for degrees of freedom on boundary nodes. To apply PBCs, it requires periodic structures. Usually, real microstructures possess randomness, i.e., no periodicity of the structure. In simple cases, as Figure 8a, artificial modification might be used to obtain the periodicity. Figure 8e is a special periodic structure obtained by adding a border of Figure $8 b$. For Figure $8 \mathrm{e}$, the PBCs can be used, but not for Figure 8b. In order to find out a better way of FE prediction, it would be beneficial to compare the inaccuracy introduced by this artificial modification of the real microstructure and by the strong constraints of homogeneous BCs. 


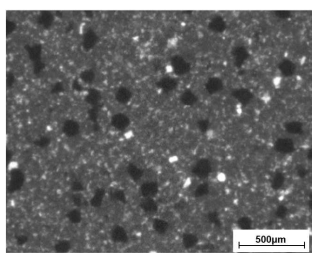

(a)

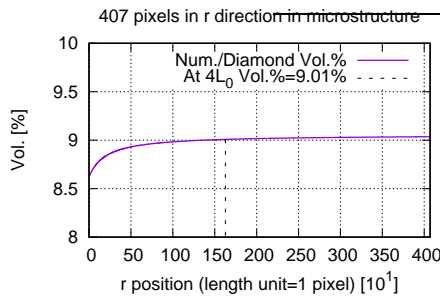

(d)

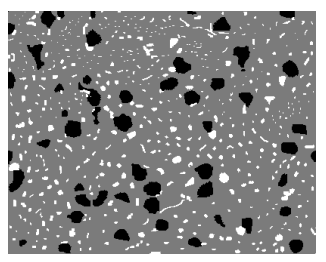

(b)

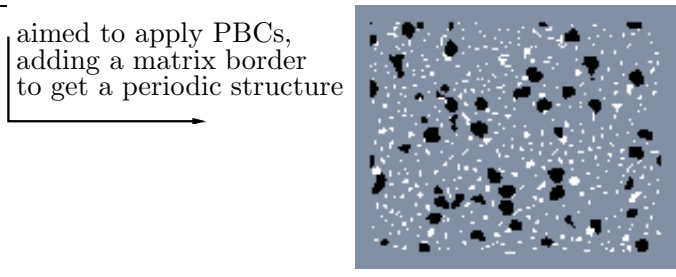

(e)

Figure 8. Co/WC/diamond (volume ratio: 90:5:5) metal matrix composites (MMCs), a kind of tool material [39,40]: (a) an image to show a microstructure cut-out with 9.06 vol.\% diamonds; (b) the same microstructure as given in (a) with the pixel resolution of $407 \times 322$ after the pixel handling; (c) identified boundary pixels of 54 diamond particles, where individual particles are assigned with different pixel color numbers; (d) the diamond phase volume fraction in an axisymmetric simulation converges to its 2D area fraction; (e) an image obtained by adding a border for (b) and suitable for application of PBCs.

It is of technological and research interest to study metal alloys with microstructures consisting of two ductile phases, e.g., $\alpha / \beta$-titanium alloys, duplex steels, $\mathrm{Cu}-\mathrm{Nb}, \mathrm{Ag}-\mathrm{Ni}$, etc. [43]. Thereby, properties of crystallites in both phases will be "combined together" to determine the macroscopic properties of the mixture. The individual properties of each phase will be changed due to the presence of the other phase. The physical and mechanical mechanisms for the aforementioned two cases are of interest for the processing of materials and for the prediction of the deformation behavior. Commentz et al. [43] experimentally and numerically studied the micro texture evolution and global stressstrain behavior of $\alpha-\mathrm{Fe} / \mathrm{Cu}$ MMCs according to $\alpha$-Fe:Cu volume fractions, where both phases are polycrystalline. Their numerical study is based on a viscoplastic self-consistent (VPSC) model, which can be categorized into a physical approach. Schneider et al. $[7,16]$ numerically studied the texture evolution by using FE simulations with an elasto-viscoplastic material model in a crystal plasticity approach, i.e., an approach by using theories of continuum mechanics. After meshing, the applied real microstructure has about 23 vol. $\% \alpha$-Fe grains (element volume) for an Fe17-Cu83 (Fe17 vol.\%-Cu83 vol.\%) MMC [7]. Figure 9a presents a microstructure cut-out of Fe17-Cu83 MMC [43] and Figure 9b is the same one with a better pixel resolution but a slightly smaller size [7,44]. Based on the morphologies given in Figure 9a,b, Figure 9c with the pixel resolution of $242 \times 301$ is obtained after the pixel handling [7], where $\alpha$-Fe has an area fraction of $23.49 \%$. By an artificial and manual alteration of polycrystalline $\alpha$-Fe into clusters with the same pixel color number, i.e., not polycrystalline $\alpha$-Fe anymore (resulted image resembles Figure $9 \mathrm{f}$ and will not be shown here). According to the structure position in the $r$ direction in Figure 3 for an axisymmetric analysis, Figure 9d illustrates the volume fraction convergence of the $\alpha$-Fe phase to its $2 \mathrm{D}$ area fraction. In Figure $9 \mathrm{~d}$, the length of one pixel is used as a unit length. At the position with $\mathrm{r}=4 L_{0}=4 \times 242=968$ unit lengths, the $\alpha$-Fe volume fraction is $23.46 \%$. Figure 9e,f illustrate the identified boundary pixels of individual $\alpha$-Fe clusters assigned with different pixel color numbers and the microstructure with 17 vol. $\% \alpha-\mathrm{Fe}$, i.e., real one, after the BPCA process. For meshing, the originally polycrystalline $\alpha$-Fe phase is needed, which is given through overlapping Figure $9 \mathrm{f}$ by Figure $9 \mathrm{c}$ for the $\alpha$-Fe phase. The resulted microstructure with polycrystalline grains for both phases is demonstrated in Figure 9g, where the colored pixels can be easily assigned during 
the overlapping by the code. The small $\alpha$-Fe grain enclosed by a red circle in Figure 9c becomes tiny after the application of the BPCA procedure (Figure 9f,g). It is user dependent, whether such a tiny grain should be meshed, since for both cases the whole calculation amount will be acceptable in a 2D or axisymmetric simulation. By using Figure 9c,g, FE predictions are compared for the homogenized stress-strain curve, the texture and local strain distribution. After meshing, the volume fraction of the $\alpha$-Fe phase is $17.31 \mathrm{vol} . \%$. It means that the volume fraction difference is $0.3 \mathrm{vol} . \%$ before (pixel) and after meshing (elements). The geometrical adaptive meshing for the region marked by a red rectangle with dashed lines in Figure 9g is presented in Figure 9h (meshing software [45]). It is worth mentioning that from Figure $9 \mathrm{c}$ to the geometrical adaptive meshing for the whole structure took only a few hours. The $\alpha$-Fe vol.\% (element volume) has a deviation less than $0.5 \%$, compared to the real one (pixel volume). By finely meshed structures, this value can be closer to the real one and the FE simulation can still properly run, since the total number of elements in an axisymmetric or 2D meshing is still within the calculation capacity, while it often exceeds the calculation capacity for 3D cases.

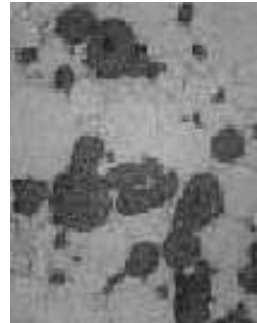

(a)

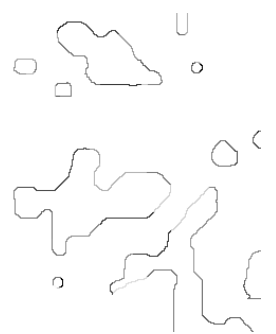

(e)

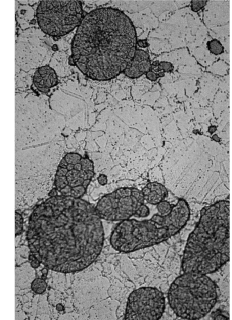

(b)

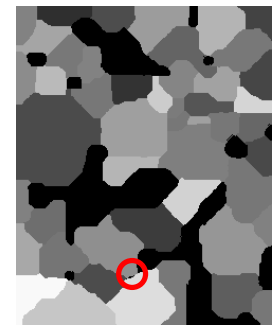

(f)

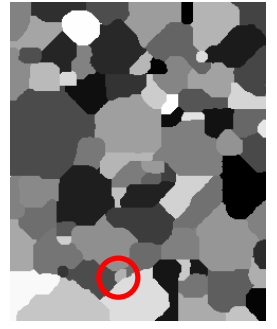

(c)

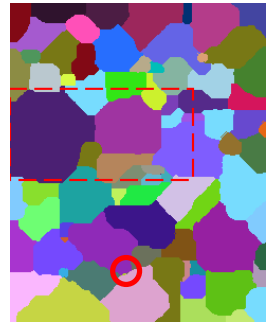

(g)

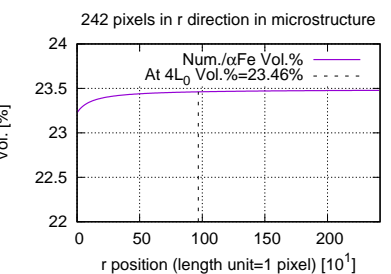

(d)

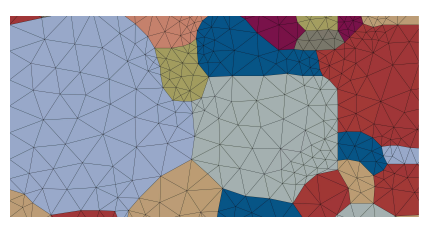

(h)

Figure 9. $\alpha$-Fe17-Cu83 polycrystalline MMC: (a) a microstructure cut-out with the darker phase presenting $\alpha$-Fe [43]; (b) nearly the same microstructure cut-out as (a) with a better pixel resolution and a slightly smaller size [7,44]; (c) after pixel handling, the same microstructure with polycrystalline $\alpha$-Fe and $\mathrm{Cu}$ grains as given in (a) with a pixel resolution of $242 \times 301$ [7]; (d) the $\alpha$-Fe phase volume fraction in an axisymmetric analysis converges to its $2 \mathrm{D}$ area fraction; (e) identified boundary pixels of individual $\alpha$-Fe clusters assigned with different pixel color numbers; (f) after the boundary pixel color alteration (BPCA), the microstructure with 17 vol. $\% \alpha$-Fe, i.e., the real one; (g) through overlapping (f) by (c) for $\alpha$-Fe phase, resulted in polycrystalline $\alpha$-Fe phase; (h) a cut-out of the geometrical adaptive meshing for the red rectangle with dashed lines in $(\mathrm{g})$.

Austenitic-ferritic steel is often used in high temperature environments. For such materials, chromium oxide scale is widely used as a protection film to resist high-temperature oxidation [46], where "scale" means an oxidized film. Aluminum oxide scale exhibits substantially greater thermodynamic stability than chromium oxide scale in many aggressive environments. For the $\mathrm{Al}$ alloyed austenitic steels, the volume fraction of the austenitic phase and of the ferritic phase is significantly influenced by the amount of alloyed $\mathrm{Al}$ elements, which is a kind of typical ferritic forming (chemical) elements. Unlike MMCs, e.g., $\mathrm{Ag} / 17 \mathrm{vol} \% \mathrm{SnO}_{2}$ and $\alpha$-Fe17-Cu83 mentioned above, the exact austenitic-ferritic volume ratio on the microscale cannot be accurately determined by the weight or volume ratios of alloyed chemical elements. Figure 10a presents a microstructure cut-out of an 
austenitic-ferritic steel with the austenitic phase shown in lighter color. After the pixel handling (Figure $10 \mathrm{~b}$ with a pixel resolution of $320 \times 240$ ), the ferritic phase has an area fraction of $21.70 \%$. In Figure 10c, the ferritic phase volume fraction, which starts with the value of $23.43 \%$ and ends at $21.80 \%$, converges to its $2 \mathrm{D}$ area fraction of $21.70 \%$ in an axisymmetric analysis. At $\mathrm{r}=4 L_{0}=960 \mu \mathrm{m}$, the austenitic phase area fraction is $21.80 \%$. Figure 10d presents the identified boundary pixels of the austenitic phase in Figure 10b. To show the phase volume convergence in an axisymmetric analysis, all the above three numerical calculations, Figures $3 c, 8 d$, and $9 d$, present the case that the original phase volume fraction is higher than the real one. For Figure 10b, it is assumed that the desired volume fraction of the austenitic phase is higher than the one in the original microstructure. In Figure 10e, the austenitic phase amounts to $30.10 \%$, i.e., a dual-phase steel (austenitic vol.\% no less than $30 \%$ ). To illustrate that the original morphologies are maintained, Figure 10f shows the overlapping result of Figure 10d (in red) and Figure 10e (in blue). It means that our BPCA method can improve the real microstructure representativeness, as well as can maintain the main characteristics of the original morphology.

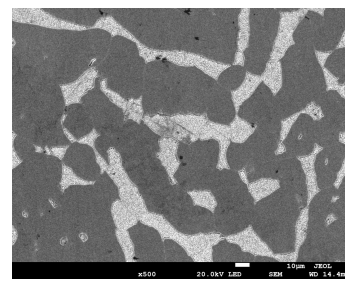

(a)

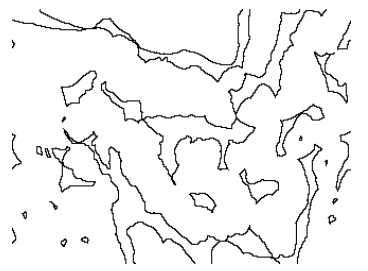

(d)

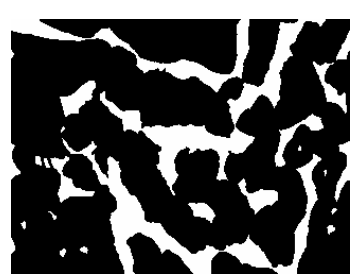

(b)

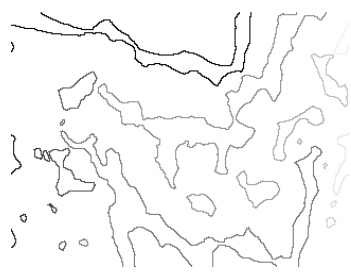

(e)

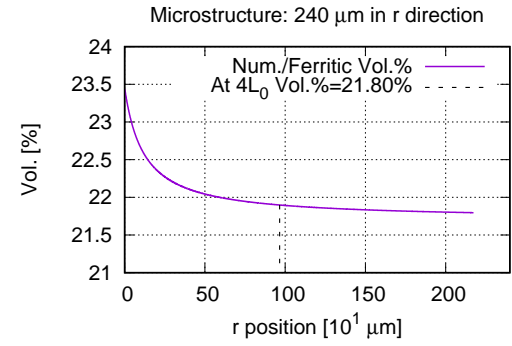

(c)

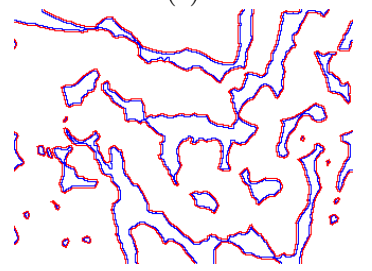

(f)

Figure 10. Two-phase steel: (a) a microstructure cut-out with the austenitic phase shown in lighter color; (b) after pixel handling, the same microstructure as given in (a) with $21.70 \%$ austenitic phase; (c) the austenitic phase volume fraction converges to its 2D area fraction in an axisymmetric analysis; (d) identified boundary pixels of the austenitic phase in (b); (e) after the boundary pixel color alteration (BPCA), the identified boundary pixels of the austenitic phase with $30.10 \mathrm{vol} \%$ austenitic phase (dual-phase steel now); (f) the original morphologies are maintained by using our BPCA method, which is shown by overlapping $(\mathbf{d}, \mathbf{e})$.

Figures $3 \mathrm{~d}, 8 \mathrm{~d}, 9 \mathrm{~d}$, and $10 \mathrm{c}$ all show the phase volume fraction (axisymmetry) convergence to its 2D area fraction. In Figures $3 \mathrm{~d}$ and $10 \mathrm{c}$, it starts with a value higher than the $2 \mathrm{D}$ area fraction, i.e., up-to-down convergence, while it is down-to-up in Figures $8 \mathrm{~d}$ and $9 \mathrm{~d}$. This should be individual local morphology and phase/grain/particle distribution dependent. The former (Figures 3d and 10c) corresponds to the case I in Appendix A Figure A1, while the latter (Figures 8d and 9d) to case II in Appendix A Figure A1.

\section{Boundary Conditions}

The current work concentrates on the application of real microstructures with rectangular shapes. For micromechanical FE simulations in one or multi-scale(s), the BCs must be considered and they, more or less, always cause disturbances for the calculated material behavior, i.e., cause inaccuracies of the predicted results. Such artificial boundary effects are introduced by materials outside the applied geometry. Two types of BCs, i.e., homogeneous and periodic ones, are often applied. The homogeneous BCs due to their strong 
constraints for the degree of freedom of boundary nodes introduce more inaccuracy than the periodic BCs. To minimize the negative influence of BCs on the calculated results, periodic $\mathrm{BC}$ are preferred, which requires periodic structures. In many cases, a real microstructure cut-out illustrates randomness, i.e., no presence of a periodic microstructure. To solve this geometrical problem, it can be considered to apply simultaneous two-scale FE simulations, as shown in Figure 4, in which no BCs are necessary anymore for the microstructure. It means that the $\mathrm{BCs}$ are applied on the macrostructure. If the emphasis of the FE simulation is put on the micromechanical deformation behavior, the $J_{2}$ theory can be used for the homogenization of material properties for the transition zone and the macrostructure. The transition zone is taken as the meshing zone, which connects the meshing on the micro and the one on the macro level. In many cases, it is a major task to calculate the stress-strain relations for FE simulations. If so, constraints for the other one (stress or strain) should be also considered, when constraints are set to one of them [47]. Taking strain controlled loading as an example, periodic BCs require periodic structures and anti-periodic conditions for corresponding stresses, e.g., as shown in Schneider et al. [7] and Fritzen et al. [48]. For special cases, e.g., as shown in Figure 8a, artificial periodic structure, adding a matrix border might be used, if this modification causes less disturbances for the calculated results than homogeneous BCs. Figure 8e illustrates the modified real microstructure, which is periodic and obtained based on Figure 8b. Even though Figure 8e is a special case, $\mathrm{PBCs}$ are applicable. Recently and based on measured in-situ 2D/3D local strain maps during the loading, real boundary conditions can be applied in FE simulations with real microstructures. But such experimental strain maps are only accessible for a few users currently.

\section{Improved FE Results by Applying the BPCA Method}

To prove the applicability of our BPCA method, FE predicted results for the polycrystalline $\alpha$-Fe17-Cu83 composite are compared by using a real microstructure (Figure 9c) and its modified one (Figure 9g). Figure 11a with $34 \alpha$-Fe and $47 \mathrm{Cu}$ grains (meshed by Reference [49]) illustrates the meshing for the aforementioned original real microstructure with about 23 vol. $\% \alpha$-Fe phase [7] and Figure 11b with $33 \alpha$-Fe and $47 \mathrm{Cu}$ grains (meshed by Reference [45]) for the modified one with 17 vol. $\% \alpha$-Fe phase.

It is possible to mesh the tiny $\alpha$-Fe grain enclosed by a red circle in Figure $9 \mathrm{~g}$ after the modification, where its original one is marked in Figures 9c and 11a. Here, it is intended that this $\alpha$-Fe grain is not meshed, i.e., disappeared in Figure 11b. It is aimed to present that the influence caused by one missing grain is negligible small for the FE results. The homogenized stress-strain flow curve is shown in Figure 12a for the compressive loading until 80\% global true strain. It is obvious that the modified real microstructure results in the better one, since the original structure has a higher global stress than the experimental one [7]. It is pointed out that the curves in Figure 12a are not comparable with the experimental one, since the initial grain orientations are far too less. The microscopic stress-strain behavior can be modified by the anisotropy of the grain orientation distribution. Figure $12 \mathrm{~b}$ presents this influence of the initial grain orientation on the stress, which is predicted by a Taylor model for the pure $\mathrm{Cu}[16,43]$. The curve marked as "mean" in Figure 12b is the homogenized one from 1000 grains and is comparable with the experimental curve, i.e., other curves in Figure $12 \mathrm{~b}$ are not. For a comparable one, the total number of grain orientations should be representative enough. In Schneider et al. [7], the numerical global stress is obtained from $34 \times 18=612 \alpha$-Fe grains and $47 \times 18=846 \mathrm{Cu}$ grains. The curves in Figure 12a are still meaningful, since for other grain orientations the same tendency will be shown. As a final result, the homogenized stress comparable with measured data will also be lower (nearer to the reality) than the one predicted by the original structure. 


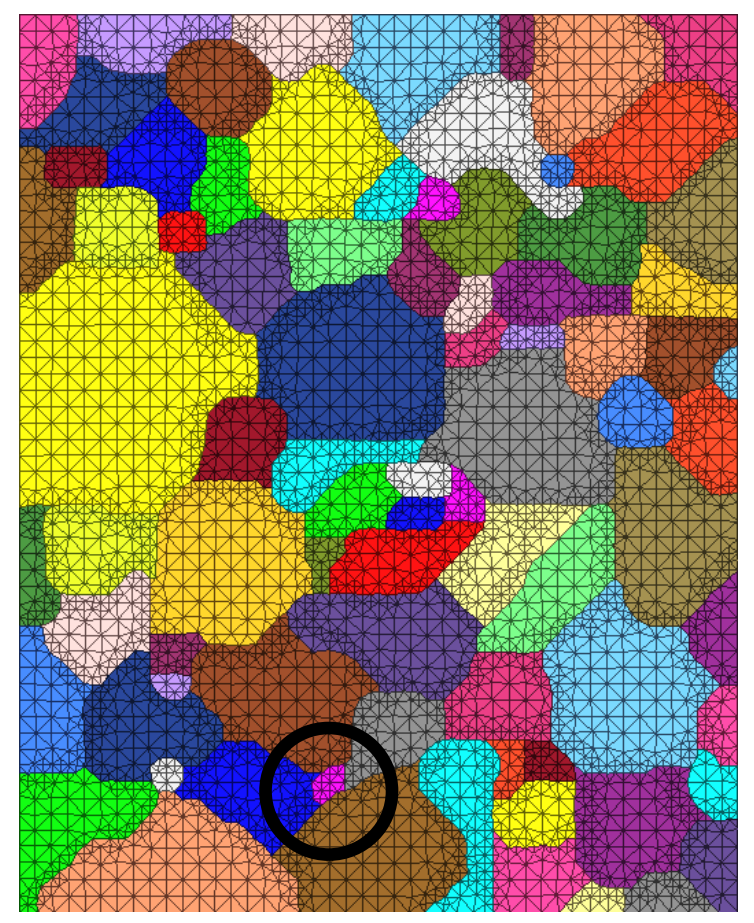

(a)

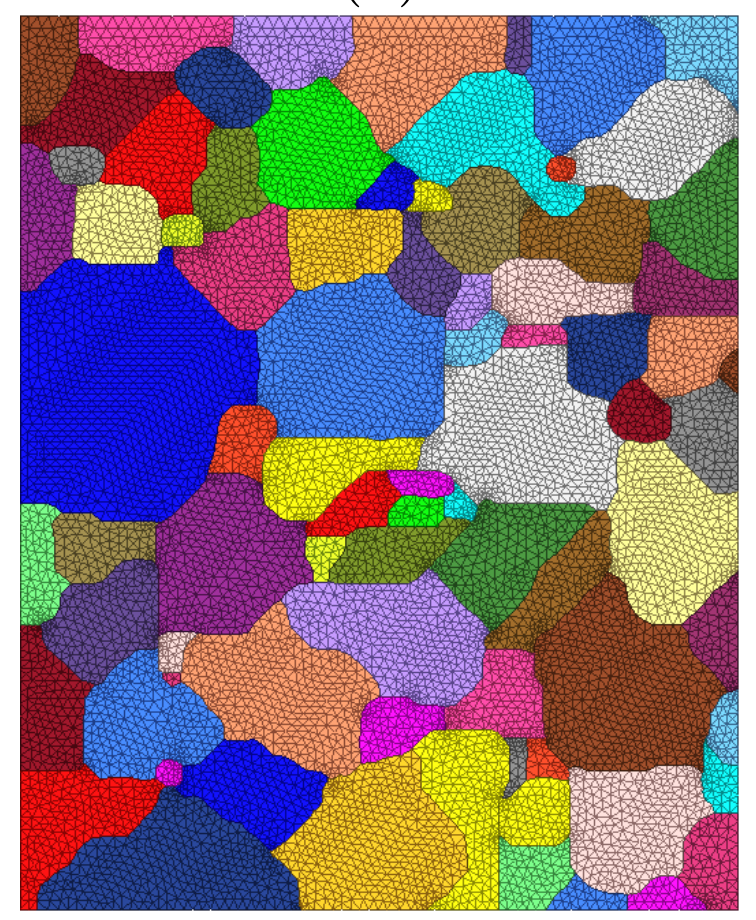

(b)

Figure 11. Meshed structures for the $\alpha$-Fe17-Cu83 polycrystalline composite: (a) original image with 23 vol. $\% \alpha$-Fe (Figure 9a) [49]; (b) modified image with 17 vol.\% $\alpha$-Fe (Figure 9g) [45]. 


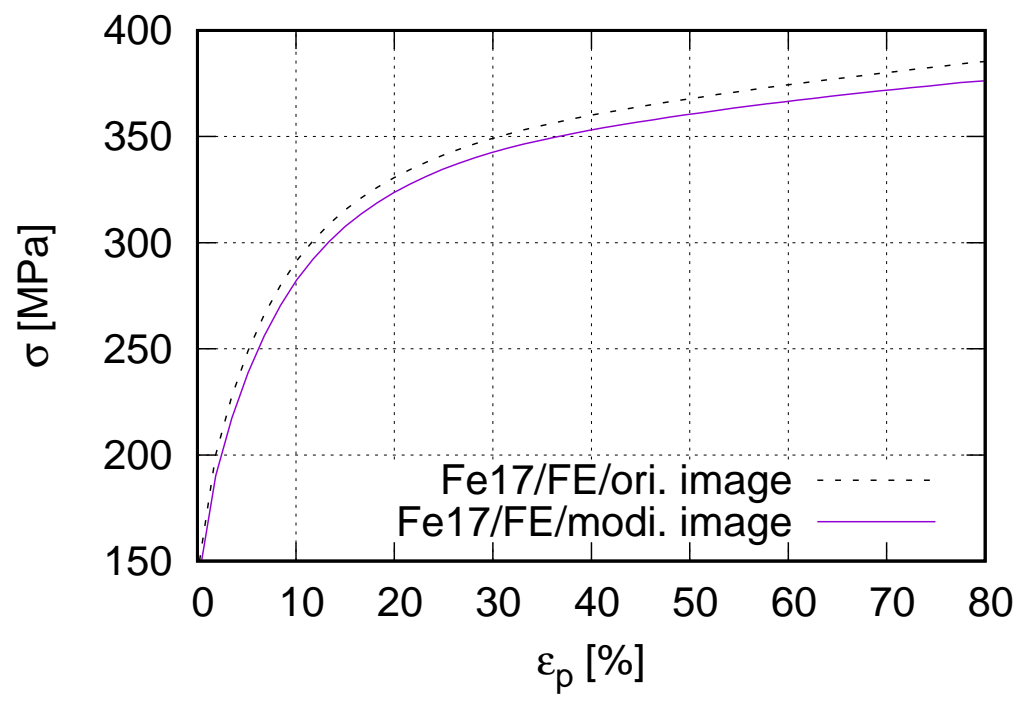

(a)

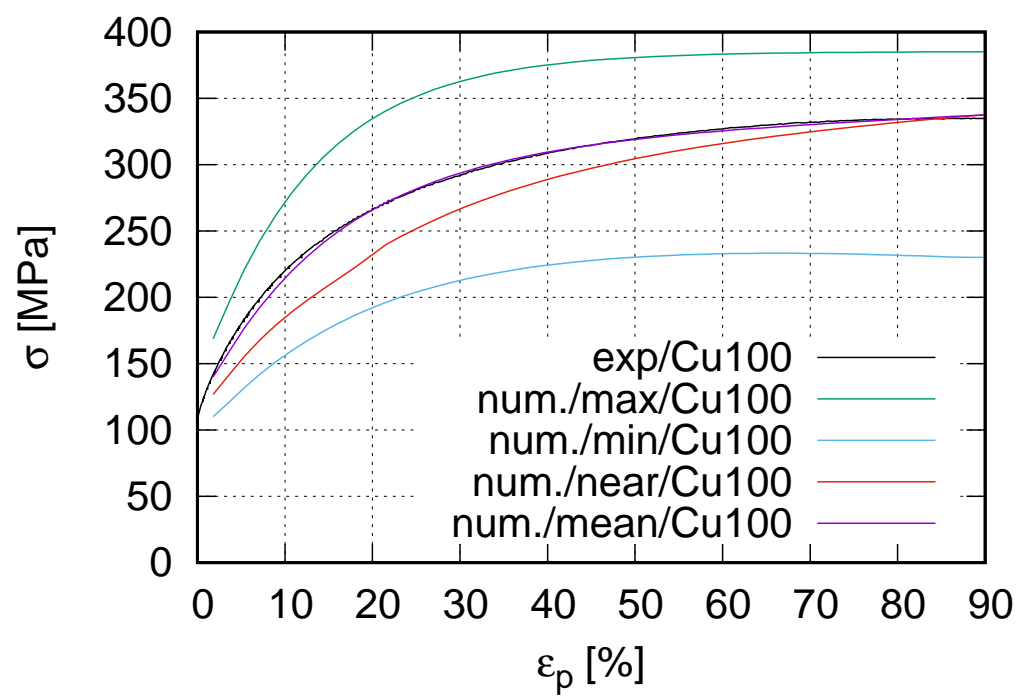

(b)

Figure 12. (a) Homogenized FE stress-strain flow curves for the $\alpha$-Fe17-Cu83 polycrystalline composite under compressive loading by using original image with $23 \mathrm{vol} . \% \alpha$-Fe and its modified one with 17 vol. $\% \alpha-F e ;(b)$ the grain orientation influence on the predicted stress-strain curves (Taylor model) by taking polycrystalline $\mathrm{Cu} 100$ (pure $\mathrm{Cu}$ ) as an example.

Figure 13a,b demonstrate the von Mises stress distribution at $80 \%$ global compressive strain, where the difference of the stress pattern is taken as being negligible small. The standard inverse pole figures obtained by applying the original microstructure are given in Figure $14 \mathrm{a}, \mathrm{b}$ for the $\mathrm{Cu}$ and $\alpha$-Fe phase, respectively. Analogously, Figure 14c,d are obtained from the modified structure. The non-identical initial grain orientations are $34,47,33$, and 47 for Figure 14a-d, respectively. Both FE results predicted by the original and modified microstructure illustrate the correct inverse pole figures. The experimental results can be referred to Schneider et al. [7]. The inverse pole figures in Figure 14a-d are not comparable with the measured ones. The reason is the same as mentioned above for Figure 12a. 


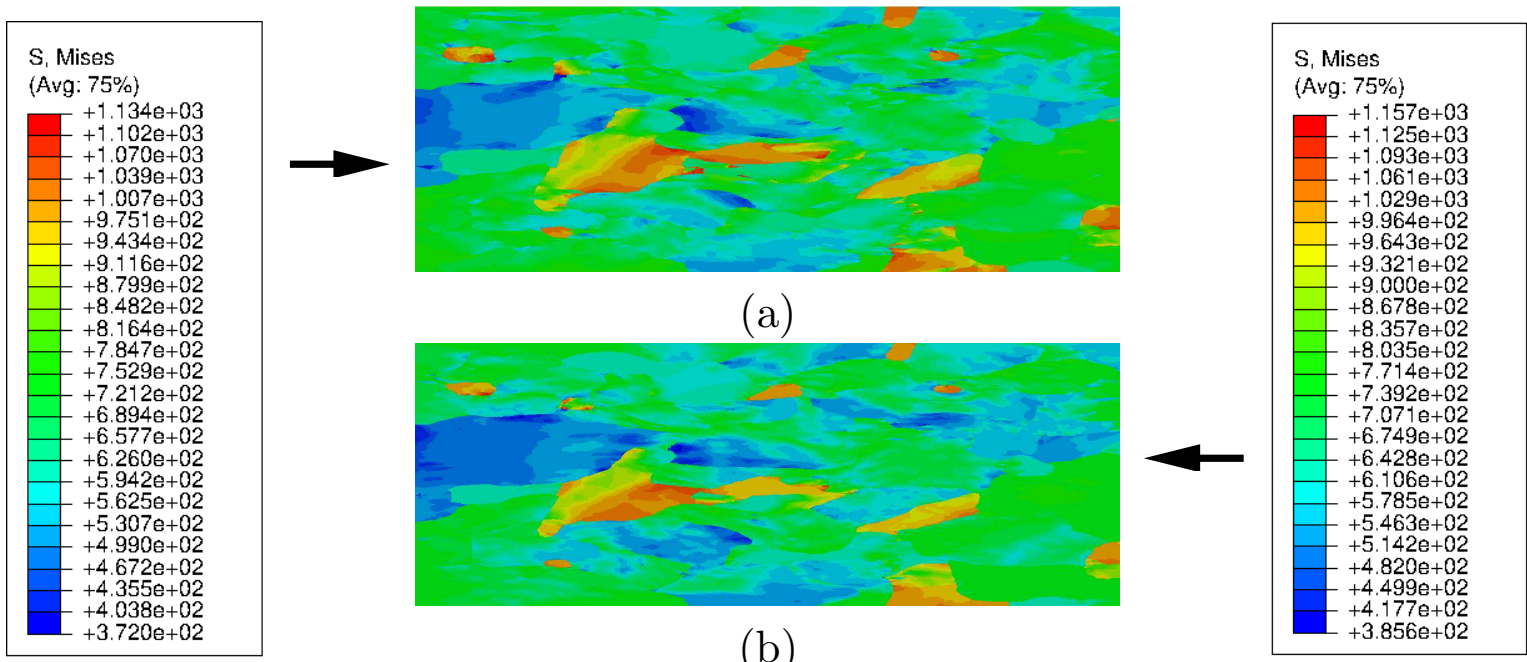

Figure 13. Stress distribution (von Mises) at $80 \%$ global compressive true strain for $\alpha$-Fe17-Cu83 polycrystalline composite: (a) original image with 23 vol. $\% \alpha$-Fe corresponding meshing given in Figure $11 \mathrm{a}$; (b) modified image with 17 vol. $\% \alpha$-Fe corresponding meshing given in Figure $11 \mathrm{~b}$.

\section{Cu phase}
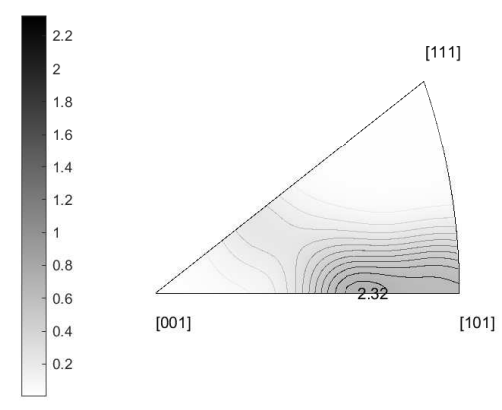

(a)

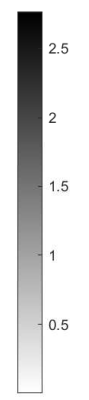

Fe phase
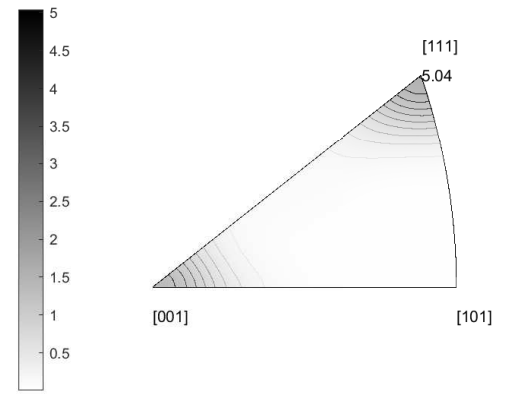

(b)

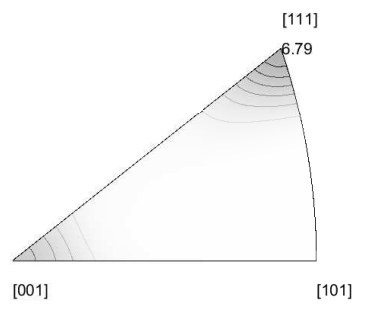

(d)

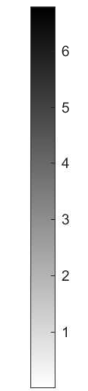

(c)

Figure 14. Standard inverse pole figure at $80 \%$ global compressive true strain for $\alpha$-Fe17-Cu83 polycrystalline composite: by using original image with 23 vol.\% $\alpha$-Fe: (a) Cu phase; (b) Fe phase; by using modified image with 17 vol. $\% \alpha$-Fe: (c) Cu phase; (d) Fe phase.

From FE results in Figures 12-14, the simulation applied with a modified real microstructure (to match real phase composition) results in: (i) a better homogenized stress flow behavior, (ii) a well comparable strain distribution with the one predicted with the 
original microstructure, and (iii) correct inverse pole figures for both $\alpha$-Fe and $\mathrm{Cu}$ phases. Concluded, the BPCA process advanced in this work is applicable to modify the real microstructure in order to reach the real composition.

\section{Conclusions}

In the field of FE simulation, the analyzing methods, 2D, axisymmetry (2.5D) and $3 \mathrm{D}$, have coexisted and will further coexist, i.e., no one can completely replace another. Micromechanical FE simulations are essential to investigate local material behavior and damage developments. In such simulations, the application of real microstructures has strong advantages. Due to the limited size, the real microstructure may not be representative enough. A less representative microstructure quite possibly leads to FE results that may not reveal the reality. The current work presents a numerical method, "boundary pixel color alteration (BPCA)" process, to modify the real microstructure cut-outs. To test the functionality of the BPCA process, the real volume/area fraction of a given phase is achieved for four 2D real microstructural cut-outs. An improvement of the predicted FE result is presented for the application of a modified microstructure (with a higher representativeness) compared to the original one. A 2D image can be used in both 2D and axisymmetric FE simulations. Based on the achievements, the following conclusions can be drawn:

- The advanced numerical procedure can not only result in a microstructure with a better representativeness but also maintain the original structure pattern (morphology).

- Besides the achievement of the real phase composition, our method can also be used to obtain the desired grain/particle sizes and distributions, since all the pixels in a microstructure are taken into consideration. The pixel color alteration can be used to change the grain/particle size and their total numbers.

- Concerning the phase volume fraction in the axisymmetric (2.5D) simulation (ABAQUS), numerical calculations done by a code show that it is indifferent to the microstructure position, even though the phase volume has a strong dependence on the microstructure position. It implies that a modified real microstructure with a higher representativeness can be applied in an axisymmetric (2.5D) simulation without a significant variation of the phase volume fraction of the material.

- A mathematical proof is presented to give the condition for the aforementioned indifference of the phase volume fraction to the microstructure position: when $r>4 L_{0}$ $\left(r / L_{0}\right.$ : position/width of the microstructure in Figure A1), the volume fraction of a phase in an axisymmetric simulation already converges to its area fraction in the $2 \mathrm{D}$ microstructure.

Author Contributions: Conceptualization Y.S. and S.S.; methodology Y.S. and S.S., software usage and simulation Y.S., S.S.; code development Y.S.; experiment and data collection W.W., R.Z., W.T., Z.Z.; writing-original draft preparation, Y.S.; review and editing all authors. All authors have read and agreed to the published version of the manuscript.

Funding: This work was supported by the German Research Foundation (Deutsche Forschungsgemeinschaft, DFG) [grant numbers SCHM 746/173-1, TI 343/118-1]. The financial support is greatfully acknowledged.

Data Availability Statement: Data is contained within the article.

Acknowledgments: We thank Kranthi Chaitanya who sorted the pixel color of the real microstructure for the $\mathrm{Ag} / \mathrm{SnO}_{2}$ composite. This work was supported by the German Research Foundation (Deutsche Forschungsgemeinschaft, DFG) [grant numbers SCHM 746/173-1, TI 343/118-1]. The financial support is greatfully acknowledged.

Conflicts of Interest: The authors declare no conflict of interest. 


\section{Appendix A. A Mathematic Proof of the Phase Volume Fraction Convergence to Its 2D Area Fraction in an Axisymmetric FE Simulation}

Motivated by the numerical results shown in Figures $3 \mathrm{c}$ and $4 \mathrm{c}$, a mathematical proof is considered to denote the reason for the aforementioned position independence of a phase volume fraction in an axisymmetric (2.5D) simulation. It means the mathematical proof should clarify the condition for this position independence. Here, the particle phase is taken as an example. The particle distribution is not identical for different microstructure cutouts. It means that a random particle distribution should be used in a direct mathematical proof. Here, "direct" indicates that the 2D microstructure will be taken as it is, i.e., no modification of the microstructure is required. Such a proof is not given by the authors. A mathematical proof in an indirect way is presented below. The word "indirect" means that the distribution of the particle phase is rearranged in a given 2D microstructure, while the particle volume fraction is maintained. The basic consideration is as follows: if the minimum and the maximum value of a variable are both close to a certain fixed value, then all the possible values for this variable are also close to this fixed value. In our example, the variable is the volume fraction of the particle phase in the axisymmetric (2.5D) FE simulation, and the aforementioned fixed value is the particle area fraction " $f_{0}$ " in the $2 \mathrm{D}$ microstructure (Figure A1).

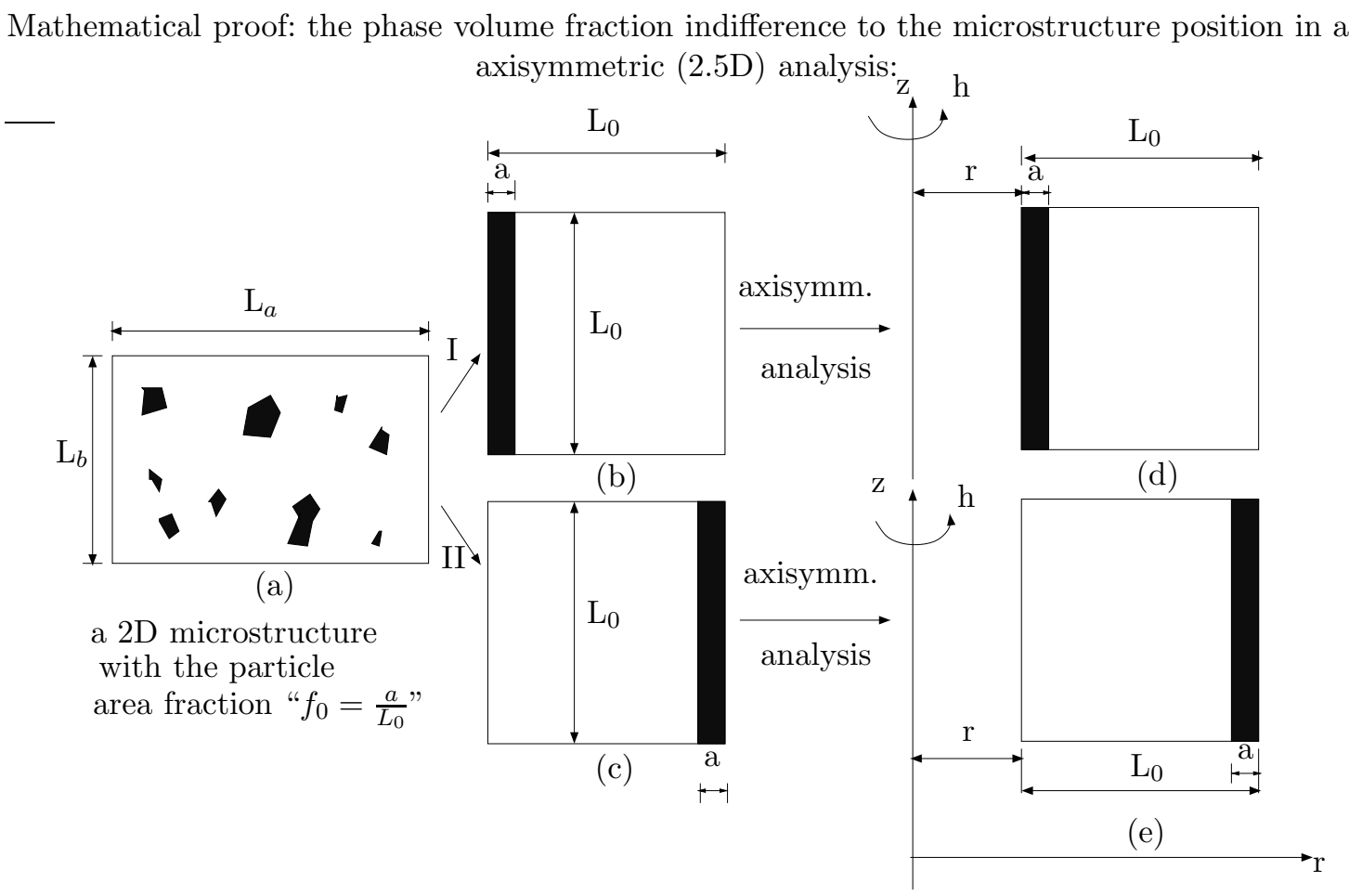

Figure A1. A schematic illustration of a phase rearrangement: (a) a sketch of a microstructure with randomly distributed particles; (b) one possible (idealized) phase distribution after the phase rearrangement; (c) another possible (idealized) phase distribution; (d) by applying (b) in an axisymmetric FE simulation, it would result in a minimum particle phase volume fraction; (e) analogously to (d), by applying (c) in an axisymmetric FE simulation, it would result in a maximum particle phase volume fraction.

Figure A1a presents a sketch of a microstructure (rectangle shape, area equals $L_{a} L_{b}$ ) with randomly distributed particles. The length and width of this microstructure are presented as " $L_{a}$ " and " $L_{b}$ ", respectively. The particle phase is given in black polygons and has an area fraction of $f_{0}$. Theoretically, $f_{0}$ can be within $(0,1)$. Practically, $f_{0}$ is in

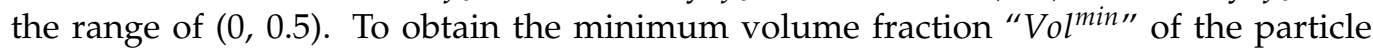
phase in an axisymmetric (2.5D) analysis, all the particles are arranged along the left edge of the 2D microstructure (Figure A1b, case I). For the maximum volume fraction 
"Vol ${ }^{\text {max", }}$ all the particles arrange along the right edge of the microstructure (Figure A1c, case II). For the sake of simplicity, a square shape with an edge length of " $L_{0}$ " is taken for the microstructure after the rearrangement of the particle phase, i.e., " $L_{a} L_{b}=L_{0}^{2}$ ". At a given position of the microstructure, "Vol total" presents the volume of the whole microstructure, " $f^{\min }$ " in Equation (A4) and " $f^{\max }$ " in Equation (A3) show the minimum and the maximum volume fraction of the particle phase, respectively. In reality, the particle phase volume fraction " $f^{\prime \prime}$ is in the range of $f^{\min }$ and $f^{\max }$, i.e., $f \in\left[f^{\min }, f^{\max }\right]$. In order to obtain the aforementioned $f^{\min }$ and $f^{\max }$, Figure A1b,c show the new distribution of the particle phase, respectively. After the rearrangement of the particle phase, $a=f_{0} L_{0}$ is valid (Figure A1b,c). In Figure A1d,e, the " $z$ ", " $h$ " and " $r$ " axes present the symmetric axis, the hoop direction and the transverse direction, respectively. The volume of the particle phase is $r$ direction/position dependent. Equation (A1) gives a formula to calculate the total volume $\mathrm{Vol}^{\text {total }}$ of the whole microstructure in an axisymmetric (2.5D) analysis (ABAQUS). Analogously, Equations (A2) and (A3) are two formulas to calculate the minimum volume $\mathrm{Vol}^{\mathrm{min}}$ and the maximum volume $\mathrm{Vol}^{\mathrm{max}}$ of the particle phase, respectively. The minimum volume fraction of the particle phase can be calculated by $f^{\text {min }}$ in Equation (A4), while the maximum one is given by $f^{\max }$ in Equation (A5).

At a prescribed position of the microstructure in an axisymmetric (2.5D) analysis (ABAQUS), the volume fraction of a considered phase in a microstructure can be calculated as follows:

Total volume of the whole microstructure (Figure A1d,e):

$$
\text { Vol }^{\text {total }}=\pi\left(r+L_{0}\right)^{2} L_{0}-\pi r^{2} L_{0}=\pi L_{0}\left(2 r L_{0}+L_{0}^{2}\right) .
$$

Possible minimum volume of the particle phase (Figure A1d):

$$
\text { Vol }^{\text {min }}=\pi(r+a)^{2} L_{0}-\pi r^{2} L_{0}=\pi L_{0}\left(2 r a+a^{2}\right) .
$$

Possible maximum volume of the particle phase (Figure A1e):

$$
\text { Vol }^{\text {max }}=\pi\left(r+L_{0}\right)^{2} L_{0}-\pi\left(r+L_{0}-a\right)^{2} L_{0}=\pi L_{0}\left(2\left(r+L_{0}\right) a-a^{2}\right) .
$$

Possible minimum volume fraction of the particle phase (Figure A1d):

$$
f^{\text {min }}=\frac{V_{\text {olmin }}^{\text {min }}}{\text { Vol total }^{\text {tol }}}=\frac{\pi L_{0}\left(2 r a+a^{2}\right)}{\pi L_{0}\left(2 r L_{0}+L_{0}^{2}\right)}=f_{0}+f_{0} \frac{f_{0} L_{0}-L_{0}}{2 r+L_{0}} .
$$

If:

$$
\begin{aligned}
& r \rightarrow 0, f^{\text {min }} \approx f_{0}+f_{0}^{2}-f_{0}=f_{0}^{2} \leq f_{0} ; \\
& r \rightarrow \infty, f^{\text {min }} \approx f_{0} ; \\
& r>4 L_{0}, f^{\text {min }}<f_{0}+f_{0} \frac{f_{0}-1}{9} \approx f_{0} .
\end{aligned}
$$

Possible maximum volume fraction of the particle phase (Figure A1e):

$$
f^{\text {max }}=\frac{V^{\text {max }}}{V_{\text {ol }}^{\text {total }}}=\frac{\pi L_{0}\left(2\left(r+L_{0}\right) a-a^{2}\right)}{\pi L_{0}\left(2 r L_{0}+L_{0}^{2}\right)}=f_{0}+f_{0} \frac{L_{0}-f_{0} L_{0}}{2 r+L_{0}} .
$$

If:

$$
\begin{aligned}
& r \rightarrow 0, f^{\max } \approx f_{0}+f_{0}-f_{0}^{2}=2 f_{0}-f_{0}^{2}, \text { i. e. } f_{0}<f^{\max }<2 f_{0} ; \\
& r \rightarrow \infty, f^{\max } \approx f_{0} ; \\
& r>4 L_{0}, f^{\max }<f_{0}+f_{0} \frac{1-f_{0}}{9} \approx f_{0} .
\end{aligned}
$$


When $r \rightarrow \infty$, both $f^{\text {min }}$ and $f^{\text {max }}$ converge to $f_{0}$. Practically, if $r>4 L_{0}, f^{\text {min }}$ and $f^{\max }$ are already very close to $f_{0}$. In our example (Figure $\left.3 \mathrm{a}\right), 0<f_{0} \approx 0.255=25.5 \%$, $L_{0} \approx 25 \mu \mathrm{m}$. At the position $r=4 L_{0} \approx 100 \mu \mathrm{m}$, the particle volume fraction $f \approx$ $25.8 \%$. This value is already very close to $f_{0}=25.5 \%$. For the FE simulation (Figure 4 ), the macrostructure has a square shape with an edge length of $42.5 \times 10^{3} \mu \mathrm{m}$. The meshing net is $680 \times 680$ elements for the macrostructure, i.e., each element with an edge length of $\frac{425,000}{680}=625 \mu \mathrm{m}$. For positions 1 and 4 in Figure $4 \mathrm{~b}$, left, the microstructure locates in the middle of the fourth element in the horizontal direction, i.e., $r>625 \times 3=1875 \mu \mathrm{m}$. In Figure $4 b$, since the microstructure is located at $r>1875>>100 \approx 4 \times L_{0} \mu \mathrm{m}$ in the macrostructure for all the positions (position 1-4), the volume fraction of the particle phase in FE simulation converges to the area fraction $f_{0}=25.5 \%$ in the microstructure cut-out (Figure 4c).

In axisymmetric (2.5D) FE simulations, the microstructure should not be positioned very closely to the symmetry axis. It is to avoid some uncertain influences from the general assumption that the symmetry axis is fixed. This means that the numerically predicted material behavior near the symmetry axis will be influenced by this assumption. The above mathematical proof (Equations (A1)-(A5)) is available for any phase in a microstructure, not only for the particle phase. In conclusion, a phase volume fraction of a microstructure in an axisymmetric (2.5D) simulation (ABAQUS) will be nearly the same as its area fraction in the 2D structure, when $r>4 L_{0}$.

\section{Appendix B. Physical Background for BPCA Method}

The presented method is basically a numerical one to improve the representativeness of a microstructure, which deals with the achievement of a real phase composition, i.e., a kind of microstructure modification. The precondition is that the microstructure to be modified is already representative to a certain extent. Our method is to solve that microstructure cut-outs used in simulations often show deviations with respect to the real phase volume ratio. These deviations negatively influence the accuracy of the numerical prediction. The physical background of the BPCA method can be related to the grain growth during the phase formation. If a local morphology, e.g., in a cut-out of a microstructure, possesses a higher volume fraction for the constituents (particles) than the real value, the growth of the matrix is suppressed by their neighbors (constituents around them), i.e., matrix "undergrowth". If the other way around is true, it means that the matrix is overgrown. Each local morphology presents a local material status. The current work concentrates on the phase volume ratio and takes a local morphology with a real phase composition as an optimum status resulted from the grain growth. The aforementioned microstructural cut-out with an "undergrown" or "overgrown" matrix demonstrates a local structure presented at a "non-optimum" material status, since the representativeness of the microstructure is not optimal in the sense of phase volume ratio.

Practically, local areas with "undergrowth" or "overgrowth" exist near to the interface between the matrix and the constituents. More directly, targets are the boundary pixels of the matrix or the particle in an image of a microstructural cut-out. Since the morphology modification to reach the real phase volume ratio merely handles the difference of the phase volume fraction between the given one and the optimum one, it implies that it refers to the end stage of the grain growth. At this time period, the phases are already formed, i.e., no new phase formation occurs any more. It also means that the main grain growth process is untouched by this BPCA method. Here, the concept of "phase" is a general one. It can be referred to the matrix or the inclusion, as well as grains, particles, or their clusters. A phase can be even a cluster composed of polycrystalline grains, e.g., the black $\alpha$-Fe clusters in Figure 9f. Simply, the phase volume fraction in the four examples (Figures 3 and 8-10) in this work are referred to matrix: inclusion volume ratio.

Figure A2 schematically demonstrates the three possible material status resulted from the grain growth for a phase. The black contour presents the "optimum" status, which possesses a real phase fraction. The red one illustrates the "overgrown" material 
status, while the blue one refers to an "undergrown" case. The former possesses a higher volume fraction than the real one, while the latter one is the other way around. Taking the "optimum" status as the starting point of analysis, when the growth of the given phase reaches this status, but develops further in all normal directions along its outer boundary and with possibly different velocities, finally, it would end in an "overgrown" status. For the "undergrown" material status, the further (virtual) growth would be in the negative normal directions. Since the volume preservation (volume constant) applies for the whole structure, when a given phase possesses an "undergrown" material status, it means that its neighboring phases possess an "overgrowth" status. Here, problems caused by the further "growth" is solved by our BPCA method (which can be taken as a virtual growing process). Connecting these two "further growth processes" to the reality: (i) the red contour is the one in the microstructure cut-out before the application of the BPCA method and the black one, the "optimum" one, corresponds to the modified microstructure cut-out after the application of BPCA method, which will be applied in the simulation; (ii) the blue contour is the starting status, i.e., before the application of BPCA method, and the black "optimum" one is the resulted contour after the application of the BPCA method.

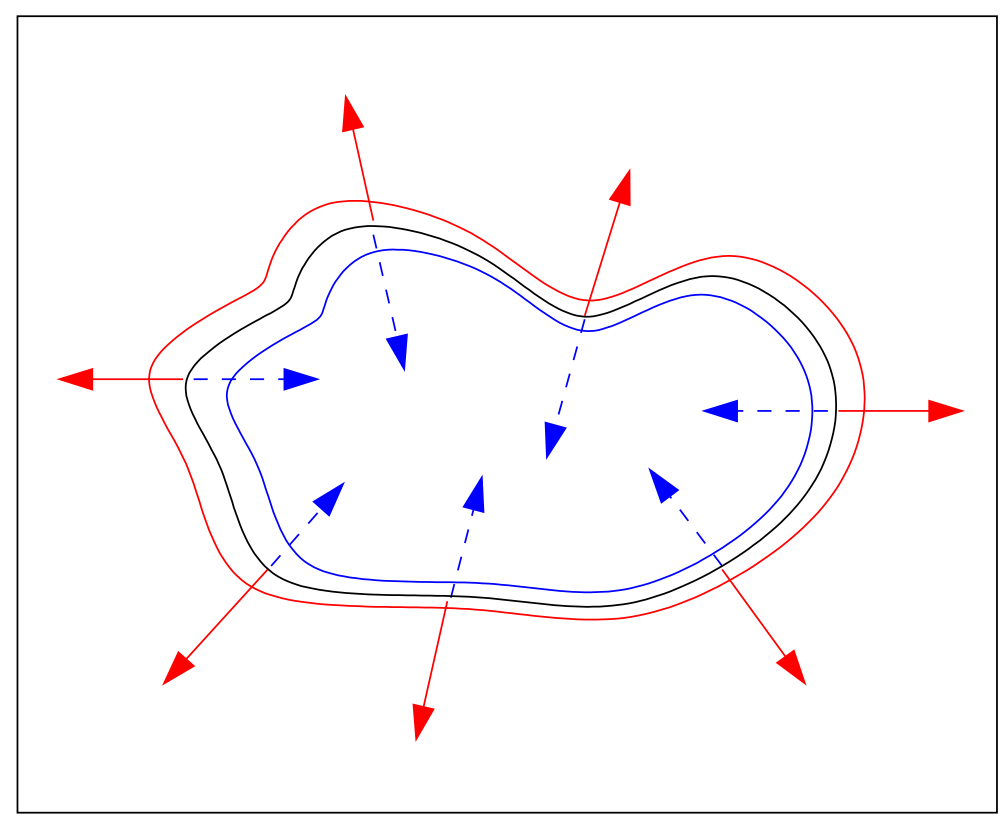

Figure A2. A schematic illustration of a phase growth, i.e., at the end stage of grain growth: black contour is assumed to be the "optimum" status, red to be "overgrown", and blue to be "undergrown" status, correspondingly.

The real microstructures are given in the form of images based on pixels, which means our BPCA method must also deal with pixels; it implies that a minimum area, which can be handled, is identical to the area of one pixel. Here, only square-pixels are considered. Figure A3 presents a virtual phase growth (microstructure modification) for the "overgrown" case to achieve the "optimum" material status. To be simple, a squareshaped phase-A is taken as an example (Figure A3a). The outer boundary (red contour) of this phase presents the phase boundary of the overgrown material status in the original microstructure. It is assumed that the area between the dashed black contour and the red one is the relevant area for the virtual grown process. This means that the phase (red contour) would grow in the negative normal direction of its outer boundary, i.e., the phase boundary will "shrink". Practically, this growth process is realized layer by layer and the minimum layer width corresponds to the edge length of a pixel. In Figure A3b,c, an easy case is taken, which means the virtual growth is accomplished inside one pixel layer. Figure A3b dislays the identified outer boundary pixels, all of which compose 
a closed loop and have other phases as neighbors. After the virtual growing process, the light brown color in Figure A3c presents the final phase geometry, which possesses the desired phase volume fraction at the "optimum" material status. Since some isolated pixels in the outer boundary layer are kept (as phase-A material points) and not continuously distributed, it means: (i) other phases did not "grow" at these material points. It implies that the growing velocity is non-identical for phases and/or at different locations; (ii) their non-continuous distribution presents the randomness of the phase growth, at least to a certain extent. Areas with other colors in Figure A3c demonstrate other phases in this material, which are neighbors to the considered phase-A. Since the whole area in Figure A3a,c, i.e., the microstructures before and after the virtual growth, are identical, it means the volume/area preserved condition is fulfilled. Since all phases have non-identical densities, the whole mass in the structure to be analyzed is not constant before and after the virtual growing process. The mass in an "optimum" material status (black contour in Figure A2) should be taken as the standard mass. It means that the mass before the virtual growing process (Figure A3a) is not the correct one, but afterwards is (Figure A3c). As a result, the virtual growing process results in a condition of the mass preservation during the grain/phase growth.

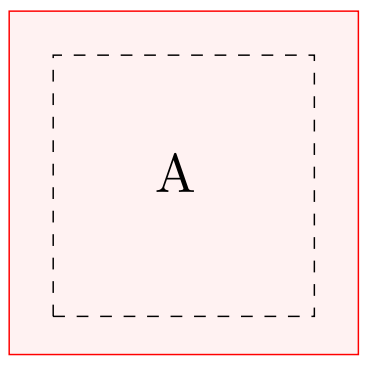

(a)

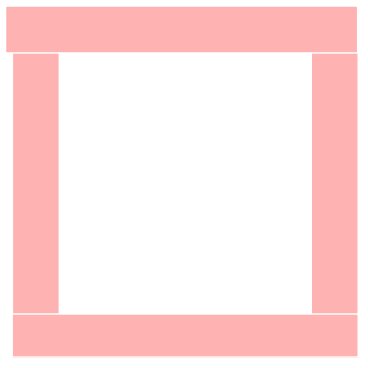

(b)

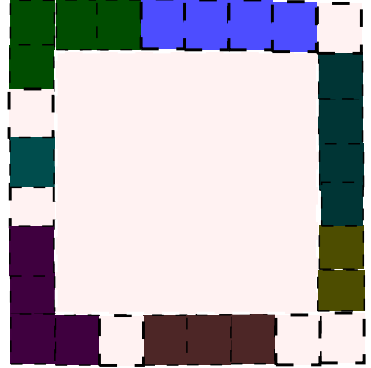

(c)

Figure A3. A schematic illustration of the virtual phase growth (microstructure modification) process for the "overgrown" case: (a) an overgrown phase in the real microstructure; (b) the area between the dashed square and the outer boundary of this phase presenting the relevant region for the virtual growth process; (c) the "optimum" phase status (light brown area) with a better representativeness of the microstructure.

\section{References}

1. Lasko, G.; Burghard, Z.; Bill, J.; Schäfer, I.; Weber, U.; Schmauder, S. Simulation of Mechanical Properties of Bio-Inspired TiO 2 /PE Nanocomposites. Adv. Eng. Mater. 2013, 1-13. [CrossRef]

2. Konijnenberg, P.; Zaefferer, S.; Raabe, D. Assessment of geometrically necessary dislocation levels derived by 3D EBSD. Acta Mater. 2015, 99, 402-414. [CrossRef]

3. Zaafarani, N.; Raabe, D.; Singh, R.; Roters, F.; Zaefferer, S. Three-dimensional investigation of the texture and microstructure below a nanoindent in a Cu single crystal using 3D EBSD and crystal plasticity finite element simulations. Acta Mater. 2006, 54, 1863-1876. [CrossRef]

4. Loeb, A.; Ferry, M.; Bassman, L. Segmentation of 3D EBSD data for subgrain boundary identification and feature characterization. Ultramicroscopy 2016, 161, 83-89. [CrossRef] [PubMed]

5. Engler, O.; Randle, V. Introduction to Texture Analysis: Macrotexture, Microtexture and Orientation Mapping, 2nd ed.; CRC Press: Boca Raton, FL, USA, 2009.

6. Mirkhalaf, S.; Pires, F.; Simoes, R. Determination of the size of the Representative Volume Element (RVE) for the simulation of heterogeneous polymers at finite strains. Finite Elem. Anal. Des. 2016, 119, 30-44. [CrossRef]

7. Schneider, Y.; Bertram, A.; Böhlke, T.; Hartig, C. Plastic deformation behaviour of Fe-Cu composites predicted by 3D finite element simulation. Comp. Mater. Sci. 2010, 48, 456-465. [CrossRef]

8. Donegan, S.P.; Kumar, N.; Groeber, M.A. Associating local microstructure with predicted thermally-induced stress hotspots using convolutional neural networks. Mater. Charact. 2019, 158, 109960. [CrossRef]

9. Willot, F.; Gillibert, L.; Jeulin, D. Microstructure-induced hotspots in the thermal and elastic responses of granular media. Int. J. Solids Struct. 2013, 50, 1699-1709. [CrossRef] 
10. Lera, F.; Angurel, L.A.; Rojo, J.A.; Mora, M.; Recuero, S.; PArroyo, M.; Andres, N. Microstructure origin of hot spots in textured laser zone melting Bi-2212 monoliths. Supercond. Sci. Technol. 2005, 18, 1489-1495. [CrossRef]

11. Rimoli, J.J.; Gürses, E.; Oritz, M. Shock-induced subgrain microstructures as possible homogenous sources of hot spots and initiation sites in energetic polycrystals. Phys. Rev. B 2010, 81, 014112. [CrossRef]

12. Chun, S.; Roy, S.; Nguyen, Y.T.; Choi, J.B.; Udaykumar, H.S.; Baek, S.S. Deep learning for synthetic microstructure generation in a materials-by-design framework for heterogeneous energetic materials. Sci. Rep. 2020, 10, 13307. [CrossRef]

13. ABAQUS/Standard; Hibbitt, Karlsson \& Sorensen, Inc.: Providence, RI, USA, 2016.

14. Wasserbäch, W.; Skrotzki, W. Microstructure and texture development in oxide-dispersion strengthened silver rods processed by hot-extrusion. Materialia 2019, 5. [CrossRef]

15. Wasserbäch, W.; Skrotzki, W.; Chekhonin, P. Strengthening of ODS silver wires. Materialia 2020, 12. [CrossRef]

16. Schneider, Y.; Bertram, A.; Böhlke, T. Three-dimensional Simulation of Local and Global Behaviour of $\alpha$ Fe-Cu Composites under Large Plastic Deformation. Tech. Mech. 2013, 33, 34-51.

17. Glüge, R.; Böhlke, T.; Bertram, A. Texture evolution and swift effect in NaAl. Proc. Appl. Math. Mech. 2006, 6, 477-478. [CrossRef]

18. Böhlke, T.; Risy, G.; Bertram, A. Finite element simulation of metal forming operations with texture based material models. Model. Simul. Mater. Sci. Eng. 2006, 14, 365-387. [CrossRef]

19. Risy, G. Modellierung der Texturinduzierten plastischen Anisotropie auf verschiedenen Skalen. Ph.D. Thesis, Fakultät für Maschinenbau, Otto-von-Guericke-Universität, Magdeburg, Germany, 2007.

20. Schneider, Y. Simulation of the Deformation Behaviour of Two-Phase Composites. Ph.D. Thesis, Fakultät für Maschinenbau, Otto-von-Guericke-Universität, Magdeburg, Germany, 2007.

21. Wulfinghoff, S.; Böhlke, T. Gradient crystal plasticity including dislocation-based work-hardening and dislocation transport. Int. J. Plast. 2015, 69, 152-169. [CrossRef]

22. Bayerschen, E.; McBride, A.; Reddy, B.D.; Böhlke, T. Review on Slip Transmission Criteria in Experiments and Crystal Plasticity Models. J. Mater. Sci. 2016, 51, 2243-2258. [CrossRef]

23. Albiez, J.; Sprenger, I.; Seemüller, C.; Weygand, D.; Heilmaier, M.; Böhlke, T. Physically motivated model for creep of directionally solidified eutectics evaluated for the intermetallic NiAl-9Mo. Acta Mater. 2016, 110, 377-385. [CrossRef]

24. Erdle, H.; Böhlke, T. A gradient crystal plasticity theory for large deformations with a discontinuous accumulated plastic slip. Comp. Mech. 2017, 60, 923-942. [CrossRef]

25. Albiez, J.; Erdle, H.; Weygand, D.; Böhlke, T. A gradient plasticity creep model accounting for slip transfer/activation at interfaces evaluated for the intermetallic NiAl-9Mo. Int. J. Plast. 2019, 113, 291-311. [CrossRef]

26. Böhlke, T.; Haus, U.; Schulze, V. Crystallographic texture approximation by quadratic programming. Acta Mater. 2006, 54, 1359-1368. [CrossRef]

27. Böhlke, T.; Risy, G.; Bertram, A. A micro-mechanically based quadratic yield condition for textured polycrystals. ZAMM 2008, 88, 379-387. [CrossRef]

28. Bertram, A. An alternative approach to finite plasticity based on material isomorphisms. Int. J. Plast. 1999, 15, 353-374. [CrossRef]

29. Bertram, A. Elasticity and Plasticity of Large Deformations-An Introduction, 2nd ed.; Springer: Berlin/Heidelberg, Germany, 2008.

30. Lee, E. Elastic-plastic deformation at finite strains. J. Appl. Mech. 1969, 36, 1-6. [CrossRef]

31. Hutchinson, J. Bounds and self-consistent estimates for creep of polycrystalline materials. Proc. R. Soc. Lond. 1976, A348, 101-127.

32. Kocks, U.F. Laws for work-hardening and low-temperature creep. J. Eng. Mater. Technol. (ASME) 1976, 98, 76-85. [CrossRef]

33. Kocks, U.F.; Mecking, H. Physics and phenomenology of strain hardening: The FCC case. Prog. Mater. Sci. 2003, 48, 171-273. [CrossRef]

34. Hill, R. Elastic properties of reinforced solids: Some theoretical principles. J. Mech. Phys. Solids 1963, 11, 357-372. [CrossRef]

35. Schneider, Y.; Wasserbäch, W.; Schmauder, S. Experimental and Micro-Macro Integrated FE Investigation of Texture and Microstructure Evolution in Polycrystalline Ag-SnO 2 Metal Matrix Composites. Proc. Appl. Math. Mech. 2017, 17, 461-462. [CrossRef]

36. Gladkov, S.; Svendsen, B. Thermodynamic and rate variational formulation of models for inhomogeneous gradient materials with microstructure and application to phase field modeling. Acta Mech. Sin. 2015, 31, 162-172. [CrossRef]

37. Kolaska, H. Pulvertechnologiesche Wege in die Zukunft; Verlage DGM Informationsgesellschaft: Oberursel, Germany, 1995.

38. Jack, D.H. Engineering Applications of Ceramic Materials: Source Book; American Society for Metals, Materials Park: Novelty, OH, USA, 1985; pp. 147-153.

39. Crostack, H.; Nellesen, J.; Fischer, G.; Schmauder, S.; Weber, U.; Beckmann, F. Tomographic analysis and FE-simulations of MMC-microstructures under load. In Developments in X-ray Tomography V; Bonse, U., Ed.; SPIE: Bellingham, WA, USA, 2006; Volume 6318, pp. 63181A-1-63181A-12. [CrossRef]

40. Crostack, H.; Nellesen, J.; Fischer, G.; Weber, U.; Schmauder, S.; Beckmann, F. 3D Analysis of MMC microstructure and deformation by $\mu \mathrm{CT}$ and FE simulations. In Developments in X-ray Tomography VI; SPIE: Bellingham, WA, USA, 2008; Volume 7078, p. 70781I.

41. Gao, H.; Huang, Y.; Nix, W.; Hutchinson, J. Mechanism-based strain gradient plasticity—I. Theory. J. Mech. Phys. Solids 1999, 47, 1239-1263. [CrossRef]

42. Huang, Y.; Gao, H.; Nix, W.; Hutchinson, J. Mechanism-based strain gradient plasticity-II Analysis. J. Mech. Phys. Solids 2000, 48, 99-128. [CrossRef] 
43. Commentz, B.; Hartig, C.; Mecking, H. Micromechanical interaction in two-phase iron-copper polycrystals. Comp. Mater. Sci. 1999, 16, 237-247. [CrossRef]

44. Commentz, B. Plastische Verformung von Zweiphasigen Eisen-Kupfer-Verbundwerkstoffen. Ph.D. Thesis, Technische Universität Hamburg, Harburg, Germany, 2000.

45. Synopsys Inc. Simpleware ScanIP 2017. Available online: https://www.synopsys.com (accessed on 2 April 2021).

46. Wang, M.; Sun, Y.D.; Feng, J.K.; Zhang, R.Q.; Tang, R.; Zhou, Z.J. Microstructural evolution and mechanical properties of an Fe-18Ni-16Cr-4Al base alloy during aging at $950{ }^{\circ} \mathrm{C}$. Int. J. Min. Met. Mater. 2016, 23, 314-322. [CrossRef]

47. Schneider, Y.; Weber, U.; Wasserbäch, W.; Zielke, R.; Schmauder, S.; Tillmann, W. A numerical method for the generation of hierarchical Poisson Voronoi microstructure applied in micromechanical finite element simulation—Part I: Method. Comp. Mech. 2020, 66, 651-667. [CrossRef]

48. Fritzen, F.; Böhlke, T.; Schnack, E. Periodic three-dimensional mesh generation for crystalline aggregates based on Voronoi tessellations. Comput. Mech. 2009, 43, 701-713. [CrossRef]

49. NIST. OOF: Object-Oriented Finite Element Analysis of Real Material Microstructures Working Group; ppm2oof1.1.24, NIST: National Institute of Standards and Technology, 2003. Available online: http:/ / www.ctcms.nist.gov/oof (accessed on 2 April 2021). 